\title{
LONG-TIME ASYMPTOTICS FOR THE FOCUSING NLS EQUATION WITH TIME-PERIODIC BOUNDARY CONDITION ON THE HALF-LINE
}

\author{
ANNE BOUTET DE MONVEL ${ }^{*}$, ALEXANDER ITS ${ }^{\dagger}$, AND VLADIMIR KOTLYAROV ${ }^{\ddagger}$ \\ Abstract. We consider the focusing nonlinear Schrödinger equation on the quarter plane. \\ The initial data are vanishing at infinity while the boundary data are time-periodic, of the \\ form $a \mathrm{e}^{\mathrm{i} \alpha} \mathrm{e}^{2 \mathrm{i} \omega t}$. The goal of this paper is to study the asymptotic behavior of the solution \\ of this initial-boundary-value problem. The main tool is the asymptotic analysis of an \\ associated matrix Riemann-Hilbert problem. We show that for $\omega<-3 a^{2}$ the solution \\ of the IBV problem has different asymptotic behaviors in different regions. In the region \\ $x>4 b t$, where $b:=\sqrt{\left(a^{2}-\omega\right) / 2}>0$, the solution takes the form of the Zakharov-Manakov \\ vanishing asymptotics. In the region $4 b t-\frac{1}{2 a} N \log t<x<4 b t$, where $N$ is any integer, \\ the solution is like a train of asymptotic solitons. In the region $4(b-a \sqrt{2}) t<x<4 b t$ the \\ solution takes the form of a modulated elliptic wave. In the region $0<x<4(b-a \sqrt{2}) t$ the \\ solution takes the form of a plane wave.
}

\section{InTRODUCTION}

The discovery of the Lax pairs for nonlinear evolutionary equations and the inverse scattering transform method (IST) for solving initial-value problems on the whole line turn out to be very successful. This powerful method gives a huge number of very interesting results in different areas of mathematics and physics. In particular, at the beginnig of 90th a new great achievement in the further development of the IST method has been done by P. Deift and X. Zhou. It is a nonlinear steepest descent method for oscillatory matrix Riemann-Hilbert problems. With this new method it came the nice possibility to rewrite known asymptotic results for different nonlinear integrable models in a rigorous and transparent form (see $[8,11,12]$ ) and obtain numerous new significant results in the theory of completely integrable nonlinear equations, random matrix models, orthogonal polynomials, and integrable statistical mechanics.

This paper continues the study of the initial-boundary-value (IBV) problem which has been originated in [4-6], and which is related to the focusing nonlinear Schrödinger equation in the quarter plane $x>0, t>0$ with time periodic boundary data and vanishing at infinity initial condition. The resulting spectral analysis [6] allows the solution to be represented in a Riemann-Hilbert form. The initial and boundary conditions must satisfy a certain global relation constraint for the IBV problem to be well posed. One of the important advantages of this method is that we obtain the solution in a very convenient form to study its long time asymptotics. Using the Deift-Zhou steepest descent method $[8,11,12]$ for oscillatory Riemann-Hilbert problems, the long time asymptotics of several IBV problems have already been studied in $[2,13-16]$, under the assumption that the boundary values of $x=0$ vanish

Date: February 21, 2008. 
for $t \rightarrow+\infty$. To the best of our knowledge IBV problems for the NLS equation with nonvanishing boundary data have not been yet considered in the framework of the RH method. We provide an implementation of the nonlinear steepest decent method for the matrix Riemann-Hilbert problem associated to the IBV problem with simplest periodic boundary data. Fortunately, this simple case contains all novelty ingredients which are necessary to pose corresponding $\mathrm{RH}$ problem for general periodic boundary data.

The problem considered in this paper is similar, though not identical, to the shock problems arising for integrable PDEs on the whole line with two different finite-gap boundary conditions as $x \rightarrow \pm \infty$. The development of the $\mathrm{RH}$ method for these problems goes back to the works done in 80-90s by R. Bikbaev, P. Deift, V. Novokshenov, and S. Venakides. Most recently, an implementation of the RH scheme to the shock problem for the focusing nonlinear Schrödinger equation on the whole line and the evaluation of the long-time asymptotics of the corresponding solution have been performed in [7]. It is worth mentioning that our construction of phase $g$-functions is different from that in [7]. Note also that there were provided numerical simulations of the considered model. The numeric results, carried out by Chunxiong Zheng, are in good agreement with our theoretical results.

The main results of this paper were announced in [3].

1.1. We consider the following initial-boundary value problem for the focusing nonlinear Schrödinger equation:

$$
\begin{aligned}
& \mathrm{i} q_{t}+q_{x x}+2|q|^{2} q=0, \quad \text { with } x, t \in \mathbb{R}_{+}, \\
& q(x, 0)=q_{0}(x), \\
& q(0, t)=g_{0}(t)=a \mathrm{e}^{\mathrm{i} \alpha} \mathrm{e}^{2 \mathrm{i} \omega t}, \\
& q_{0}(0)=g_{0}(0)=a \mathrm{e}^{\mathrm{i} \alpha},
\end{aligned}
$$

where $q_{0}(x)$ vanishes for $x \rightarrow+\infty, a>0, \alpha$ and $\omega$ are real numbers. We suppose that the solution $q(x, t)$ of the IBV problem exists for $x, t \in \mathbb{R}_{+}$. This solution is $C^{\infty}$, continuous with all its derivatives up to the boundary $\{x=0\} \cup\{t=0\}$ of the quarter $x t$-plane and $q(x, t) \in \mathcal{S}\left(\mathbb{R}_{+}\right)$in $x$ for any fixed $t \in \mathbb{R}_{+}$. Here $\mathcal{S}\left(\mathbb{R}_{+}\right)$is the space of Schwartz functions on $\mathbb{R}_{+}$:

$$
\mathcal{S}\left(\mathbb{R}_{+}\right)=\left\{u(x) \in C^{\infty}\left(\mathbb{R}_{+}\right) \mid x^{n} u^{(m)}(x) \in L^{\infty}\left(\mathbb{R}_{+}\right) \text {for any } n, m \geq 0\right\} .
$$

We assume the initial data $q_{0}(x) \in \mathcal{S}\left(\mathbb{R}_{+}\right)$. It also worth noticing that all the considerations of this paper are actually valid if the boundary condition (1.1c) is replaced by its natural weaker version,

$$
q(0, t)=g_{0}(t)=a \mathrm{e}^{\mathrm{i} \alpha} \mathrm{e}^{2 \mathrm{i} \omega t}+v_{0}(t)
$$

with $v_{0}(t) \in \mathcal{S}\left(\mathbb{R}_{+}\right)$.

The focusing nonlinear Schrödinger equation admits [17] a Lax pair consisting of the two linear eigenvalue problems presented below - equations (1.3) and (1.4). For the study of the initial-boundary value problem (1.1) we shall use, following the methodology of [14], a 
simultaneous spectral analysis of two eigenvalue problems, one for the linear $x$-equation:

$$
\begin{aligned}
& \Phi_{x}+\mathrm{i} k \sigma_{3} \Phi=Q(x, t) \Phi, \\
& Q(x, t):=\left(\begin{array}{cc}
0 & q(x, t) \\
-\bar{q}(x, t) & 0
\end{array}\right) \\
& \sigma_{3}:=\left(\begin{array}{cc}
1 & 0 \\
0 & -1
\end{array}\right)
\end{aligned}
$$

and the other for the linear $t$-equation

$$
\begin{aligned}
& \Phi_{t}+2 \mathrm{i} k^{2} \sigma_{3} \Phi=\tilde{Q}(x, t ; k) \Phi, \\
& \tilde{Q}(x, t ; k):=2 k Q(x, t)-\mathrm{i}\left(Q^{2}(x, t)+Q_{x}(x, t)\right) \sigma_{3},
\end{aligned}
$$

where $\Phi(x, t ; k)$ is a $2 \times 2$ matrix-valued function, $k \in \mathbb{C}$. It is well-known that this system of linear equations is compatible $[1,17]$ if and only if $q(x, t)$ solves the nonlinear Schrödinger equation.

1.2. To formulate the Riemann-Hilbert problem related to the IBV problem (1.1), we need to introduce spectral functions using the initial data, and the Dirichlet and Neumann boundary data. Thus we have to make an assumption on the structure of the Dirichlet to Neumann map. For $\omega<-3 a^{2}$ we claim that this map takes the form (1.5) below.

Assumptions. We assume that the IBV problem (1.1) has a global solution $q(x, t)$, sufficiently smooth and with sufficient decay for $x \rightarrow+\infty$. We also assume that for $\omega<-3 a^{2}$ the Neumann boundary values take the form

$$
\begin{aligned}
& q_{x}(0, t)=g_{1}(t)=2 \mathrm{i} a b \mathrm{e}^{\mathrm{i} \alpha} \mathrm{e}^{2 \mathrm{i} \omega t}+v_{1}(t), \quad \text { with } v_{1}(t) \in \mathcal{S}\left(\mathbb{R}_{+}\right), \\
& b:=\sqrt{\frac{a^{2}-\omega}{2}}>0 .
\end{aligned}
$$

Note that numerical simulations provided by Chunxiong Zheng are in good agreement with this last assumption. This assumption is also supported by the asymptotic results obtained in section 5.4 (see Remark 4 at the end of section 5.4).

Remark 1. The structure of the Dirichlet to Neumann map depends essentially on the relation between the frequency $\omega$ and the amplitude $a$. An exact example in the case $\omega \geq a^{2} / 2$ shows that the condition (1.5) is no longer valid for every initial-boundary data. Indeed, it follows from this example that there is a component in the space of the data where, instead of (1.5), the relevant assumption about the behavior of $q_{x}(0, t)$ is:

$$
\begin{aligned}
& q_{x}(0, t)=2 a \hat{b} \mathrm{e}^{\mathrm{i} \alpha} \mathrm{e}^{2 \mathrm{i} \omega t}+v_{1}(t), \quad \text { with } v_{1}(t) \in \mathcal{S}\left(\mathbb{R}_{+}\right), \\
& \hat{b}:=\sqrt{\frac{\omega}{2}-\frac{a^{2}}{4}}>0 .
\end{aligned}
$$

The example is the following exact solution — stationary soliton — of the NLS equation:

$$
q(x, t)=\sqrt{2 \omega} \frac{\mathrm{e}^{\mathrm{i} \alpha} \mathrm{e}^{2 \mathrm{i} \omega t}}{\cosh \sqrt{2 \omega}\left(x-x_{0}\right)}=2 \eta \mathrm{e}^{\mathrm{i} \alpha} \frac{\mathrm{e}^{4 \mathrm{i} \eta^{2} t}}{\cosh 2 \eta\left(x-x_{0}\right)}
$$


where

$$
\eta:=\frac{1}{2} \sqrt{2 \omega}
$$

For this solution we have:

$$
q(0, t)=a \mathrm{e}^{\mathrm{i} \alpha} \mathrm{e}^{2 \mathrm{i} \omega t}
$$

and

$$
q_{x}(0, t)=4 \eta^{2} \frac{\mathrm{e}^{4 \mathrm{i} \eta^{2} t}}{\cosh 2 \eta\left(x_{0}\right)} \tanh 2 \eta x_{0} \equiv 2 a \hat{b} \mathrm{e}^{\mathrm{i} \alpha} \mathrm{e}^{2 \mathrm{i} \omega t}
$$

where

$$
\begin{aligned}
& a=\frac{2 \eta}{\cosh 2 \eta x_{0}}, \\
& \hat{b}=\eta \tanh 2 \eta x_{0} .
\end{aligned}
$$

In this case we have the relation

$$
a^{2}+4 \hat{b}^{2}=2 \omega .
$$

Thus the stationary soliton is the explicit solution of the IBV problem (1.1), satisfying (1.6), with initial data

$$
q(x, 0)=\sqrt{2 \omega} \frac{\mathrm{e}^{\mathrm{i} \alpha}}{\cosh \sqrt{2 \omega}\left(x-x_{0}\right)} .
$$

1.3. In this paper we restrict our attention to the case $\omega<-3 a^{2}$. The focusing NLS equation admits the exact solution

$$
\begin{aligned}
& q_{\mathrm{p}}(x, t)=a \mathrm{e}^{\mathrm{i} \alpha} \mathrm{e}^{2 \mathrm{i} b x+2 \mathrm{i} \omega t}, \\
& b:=\sqrt{\left(a^{2}-\omega\right) / 2}>0, \quad a>0 .
\end{aligned}
$$

In this case we have the relation

$$
a^{2}-2 b^{2}=\omega
$$

Let $\tilde{Q}_{\mathrm{p}}(t, k)=\tilde{Q}_{\mathrm{p}}(0, t, k)$ where $\tilde{Q}_{\mathrm{p}}(x, t ; k)$ is defined like $\tilde{Q}(x, t ; k)$ but starting from $q_{\mathrm{p}}(x, t)$ instead of $q(x, t)$, i.e.

$$
\tilde{Q}_{\mathrm{p}}(t, k):=2 k Q_{\mathrm{p}}(t)-\mathrm{i}\left(Q_{\mathrm{p}}^{2}(t)+\left(Q_{\mathrm{p}}\right)_{x}(t)\right) \sigma_{3}
$$

with

$$
\begin{aligned}
& Q_{\mathrm{p}}(t):=Q_{\mathrm{p}}(0, t)=\left(\begin{array}{cc}
0 & a \mathrm{e}^{\mathrm{i} \alpha} \mathrm{e}^{2 \mathrm{i} \omega t} \\
-a \mathrm{e}^{-\mathrm{i} \alpha} \mathrm{e}^{-2 \mathrm{i} \omega t} & 0
\end{array}\right), \\
& \left(Q_{\mathrm{p}}\right)_{x}(t):=\left(Q_{\mathrm{p}}\right)_{x}(0, t)=\left(\begin{array}{cc}
0 & 2 \mathrm{i} a \mathrm{e}^{\mathrm{i} \alpha} b \mathrm{e}^{2 \mathrm{i} \omega t} \\
2 \mathrm{i} a \mathrm{e}^{-\mathrm{i} \alpha} b \mathrm{e}^{-2 \mathrm{i} \omega t} & 0
\end{array}\right), \\
& Q_{\mathrm{p}}(x, t):=\left(\begin{array}{cc}
0 & q_{\mathrm{p}}(x, t) \\
-\bar{q}_{\mathrm{p}}(x, t) & 0
\end{array}\right) .
\end{aligned}
$$

Consider now the $t$-part (1.4a) of the Lax pair associated with $\tilde{Q}_{\mathrm{p}}(t)$, i.e.

$$
\Psi_{t}(t, k)+2 \mathrm{i} k^{2} \sigma_{3} \Psi(t, k)=\tilde{Q}_{\mathrm{p}}(t, k) \Psi(t, k), \quad t>0, \quad k \in \mathbb{C}
$$


where $\Psi(t, k)$ is $2 \times 2$ matrix-valued. A particular solution of (1.7) is given by

$$
\begin{aligned}
& \Psi(t, k)=\mathcal{E}(t, k) \mathrm{e}^{\mathrm{i}(\omega-\Omega(k)) \sigma_{3} t}, \\
& \mathcal{E}(t, k)=\mathrm{e}^{\mathrm{i} \omega \hat{\sigma}_{3} t} E(k):=\mathrm{e}^{\mathrm{i} \omega \sigma_{3} t} E(k) \mathrm{e}^{-\mathrm{i} \omega \sigma_{3} t}, \\
& E(k)=\frac{1}{2}\left(\begin{array}{cc}
\nu(k)+\frac{1}{\nu(k)} & \mathrm{e}^{\mathrm{i} \alpha}\left(\nu(k)-\frac{1}{\nu(k)}\right) \\
\mathrm{e}^{-\mathrm{i} \alpha}\left(\nu(k)-\frac{1}{\nu(k)}\right) & \nu(k)+\frac{1}{\nu(k)}
\end{array}\right) \\
& \Omega(k)=2(k-b) X(k) \\
& X(k)=\sqrt{(k+b)^{2}+a^{2}}, \\
& \nu(k)=\left(\frac{k+b-\mathrm{i} a}{k+b+\mathrm{i} a}\right)^{\frac{1}{4}} .
\end{aligned}
$$

We fix the branches of the square roots by their asymptotics, for $k \rightarrow \infty$ :

$$
\begin{aligned}
& X(k)=\sqrt{(k+b)^{2}+a^{2}}=k+b+\mathrm{O}\left(k^{-1}\right), \\
& \nu(k)=\left(\frac{k+b-\mathrm{i} a}{k+b+\mathrm{i} a}\right)^{\frac{1}{4}}=1-\frac{\mathrm{i} a}{2 k}+\mathrm{O}\left(k^{-2}\right)
\end{aligned}
$$

on the complex $k$-plane cut along any curve connecting the two branch points $E$ and $\bar{E}$.

In this paper we carry out the principal ingredients of the asymptotic analysis of the basic Riemann-Hilbert problem which is formulated below and whose detailed presentation is given in [6]. Under certain further technical assumptions on the Riemann-Hilbert data, we shall describe the long time asymptotics of the solution of the related IBV problem.

Notations. (1) If $\mu$ is a $2 \times 2$ matrix we denote its columns by $[\mu]_{1}$ and $[\mu]_{2}$.

(2) Let $\Phi$ be a function defined in a neighborhood of an oriented contour $\Sigma$ in the Riemann sphere $\mathbb{C} \cup\{\infty\}$ or in some Riemann surface and let $k \in \Sigma$ be a non self-crossing point. We denote by $\Phi_{+}(k)$ the boundary value of $\Phi$ at $k$ from the left side and by $\Phi_{-}(k)$ its boundary value from the right side.

\section{EigenfunCtions}

We first define the contour

$$
\Sigma:=\{k \in \mathbb{C} \mid \operatorname{Im} \Omega(k)=0\},
$$

where $\Omega(k)$ is given by (1.8d). Let us put $k_{1}=\operatorname{Re} k$ and $k_{2}=\operatorname{Im} k$. Then the equation $\operatorname{Im} \Omega(k)=0$ means

$$
\begin{aligned}
& k_{2}=0 \text { or } \\
& k_{1} k_{2}^{2}=\left(k_{1}-b\right)\left(k_{1}^{2}+b k_{1}+\frac{a^{2}}{2}\right)=\left(k_{1}-b\right)\left(k_{1}-\kappa_{-}\right)\left(k_{1}-\kappa_{+}\right) \text {with }\left|k_{1}\right| \leq|b|,
\end{aligned}
$$

where $2 b^{2}=a^{2}-\omega$. In what follows we suppose $\omega<-3 a^{2}$, i.e., $b^{2}>2 a^{2}$, and $b>0$. Therefore, $\kappa_{ \pm}$are real and

$$
\kappa_{ \pm}=-\frac{b}{2} \pm \sqrt{\frac{b^{2}}{4}-\frac{a^{2}}{2}}
$$


with $-b<\kappa_{-} \leq-b / 2 \leq \kappa_{+}<0$ (Figure 1$)$.

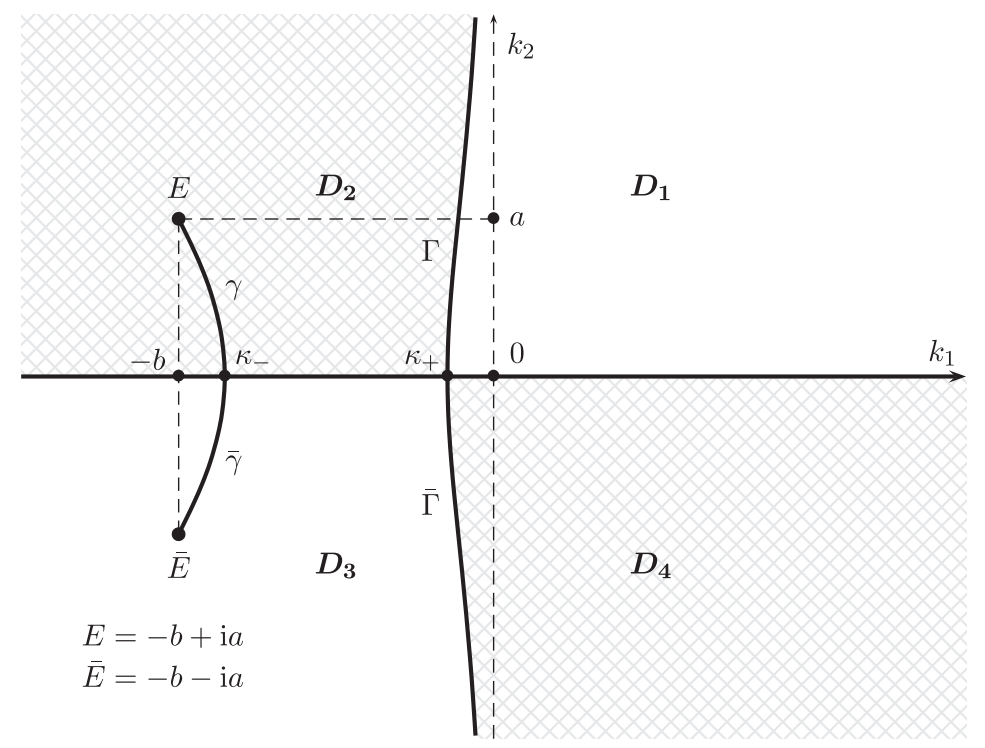

Figure 1. The domains $D_{j}$ for $b^{2}>2 a^{2}, a>0, b>0$

The contour $\Sigma$ consists of the real axis $\mathbb{R}$, the finite arc $\gamma \cup \bar{\gamma}$ whose endpoints are the branch points $E=-b+\mathrm{i} a$ and $\bar{E}=-b-\mathrm{i} a$, and the contour $\Gamma \cup \bar{\Gamma}$ :

$$
\Sigma=\mathbb{R} \cup \gamma \cup \bar{\gamma} \cup \Gamma \cup \bar{\Gamma} \text {. }
$$

The $D_{j}, j=1,2,3,4$ are the following domains:

$$
\begin{aligned}
& D_{1}:=\{k \in \mathbb{C} \mid \operatorname{Im} k>0, \operatorname{Im} \Omega(k)>0\}, \\
& D_{2}:=\{k \in \mathbb{C} \mid \operatorname{Im} k>0, \operatorname{Im} \Omega(k)<0\}, \\
& D_{3}:=\{k \in \mathbb{C} \mid \operatorname{Im} k<0, \operatorname{Im} \Omega(k)>0\}, \\
& D_{4}:=\{k \in \mathbb{C} \mid \operatorname{Im} k<0, \operatorname{Im} \Omega(k)<0\} .
\end{aligned}
$$

We also define

$$
\begin{aligned}
& \Omega_{+}:=D_{1} \cup D_{3}=\{k \in \mathbb{C} \mid \operatorname{Im} \Omega(k)>0\}, \\
& \Omega_{-}:=D_{2} \cup D_{4}=\{k \in \mathbb{C} \mid \operatorname{Im} \Omega(k)<0\} .
\end{aligned}
$$

So we obtain a partition of the complex $k$-plane $\mathbb{C}$ :

$$
D_{1} \cup D_{2} \cup D_{3} \cup D_{4} \cup \Sigma=\mathbb{C} .
$$

We assume that there exists a unique global solution $q(x, t)$ satisfying (1.1) and (1.5) and we consider the associated functions $Q(x, t)$ and $\tilde{Q}(x, t ; k)$ defined by $(1.3 \mathrm{~b})$ and $(1.4 \mathrm{~b})$, respectively. Define the $2 \times 2$ matrix-valued functions $\left\{\mu_{j}(x, t ; k)\right\}_{j=1}^{3}$ for $0<x<\infty$ and 
$0<t<\infty$, as the solutions of the following Volterra integral equations:

$$
\begin{aligned}
\mu_{3}(x, t ; k)= & I-\int_{x}^{\infty} \mathrm{e}^{\mathrm{i} k(\xi-x) \hat{\sigma}_{3}}\left(Q \mu_{3}\right)(\xi, t ; k) \mathrm{d} \xi \\
\mu_{2}(x, t ; k)= & I+\mathrm{e}^{-\mathrm{i} k x \hat{\sigma}_{3}} \int_{0}^{t} \mathrm{e}^{-2 \mathrm{i} k^{2}(t-\tau) \hat{\sigma}_{3}}\left(\tilde{Q} \mu_{2}\right)(0, \tau ; k) \mathrm{d} \tau \\
& +\int_{0}^{x} \mathrm{e}^{-\mathrm{i} k(x-\xi) \hat{\sigma}_{3}}\left(Q \mu_{2}\right)(\xi, t ; k) \mathrm{d} \xi \\
\mu_{1}(x, t ; k)= & \mathrm{e}^{-\mathrm{i} k x \hat{\sigma}_{3}+\mathrm{i} \omega t \hat{\sigma}_{3}} E(k) \\
& +\mathrm{e}^{-\mathrm{i} k x \hat{\sigma}_{3}} \mathcal{E}(t, k) \int_{\infty}^{t} \mathrm{e}^{\mathrm{i}[\omega-\Omega(k)](t-\tau) \hat{\sigma}_{3}} \mathcal{E}^{-1}(\tau ; k) \tilde{Q}_{0}(\tau ; k) \mu_{1}(0, \tau ; k) \mathrm{d} \tau \\
& +\int_{0}^{x} \mathrm{e}^{-\mathrm{i} k(x-\xi) \hat{\sigma}_{3}}\left(Q \mu_{1}\right)(\xi, t ; k) \mathrm{d} \xi
\end{aligned}
$$

where $E(k), \mathcal{E}(t, k)$, and $\Omega(k)$ are defined by $(1.8 \mathrm{~b}),(1.8 \mathrm{c})$, and (1.8d) respectively, and

$$
\tilde{Q}_{0}(t ; k):=\tilde{Q}(0, t ; k)-\tilde{Q}_{\mathrm{p}}(t ; k) .
$$

Proposition 1. The $2 \times 2$ matrices $\left\{\mu_{j}(x, t ; k)\right\}_{j=1}^{3}$ have the following properties:

(i) For $j=1,2,3$ :

$$
\operatorname{det} \mu_{j}(x, t ; k) \equiv 1 \text {. }
$$

(ii) The functions $\left\{\Phi_{j}\right\}_{j=1}^{3}$ defined by

$$
\begin{aligned}
& \Phi_{1}(x, t ; k):=\mu_{1}(x, t ; k) \mathrm{e}^{-\mathrm{i} k x \sigma_{3}+\mathrm{i}[\omega-\Omega(k)] t \sigma_{3}}, \\
& \Phi_{j}(x, t ; k):=\mu_{j}(x, t ; k) \mathrm{e}^{-\mathrm{i} k x \sigma_{3}-2 \mathrm{i} k^{2} t \sigma_{3}}, \quad j=2,3
\end{aligned}
$$

satisfy the Lax pair (1.3)-(1.4).

(iii) For $j=1,2,3$ :

$$
\mu_{j}(x, t ; k)=I+\mathrm{O}\left(k^{-1}\right), \quad k \rightarrow \infty, \quad \operatorname{Im} k=0 .
$$

(iv) Near $k=-b \pm \mathrm{i} a$, the matrix $\mu_{1}(x, t ; k)$ exhibits inverse fourth-root singularities like those the matrix $E(k)$ has.

(v) The matrix $\mu_{1}(x, t ; k)$ has different boundary values along a cut $\gamma$ connecting the two points $k=-b \pm \mathrm{i} a$, which are the branch points of the function $X(k)$.

(vi) The matrix $\mu_{2}(x, t ; k)$ is entire in $k \in \mathbb{C}$. Furthermore

$$
\mu_{1}=\left(\begin{array}{ll}
\mu_{1}^{(2)} & \mu_{1}^{(3)}
\end{array}\right), \quad \mu_{2}=\left(\begin{array}{ll}
\mu_{2}^{(1)} & \mu_{2}^{(4)}
\end{array}\right), \quad \mu_{3}=\left(\begin{array}{ll}
\mu_{3}^{(34)} & \mu_{3}^{(12)}
\end{array}\right),
$$

where
(a) $\mu_{1}^{(2)}$ means that the first column vector $\left[\mu_{1}(x, t ; k)\right]_{1}$ is bounded and analytic in $D_{2}$,
(b) $\mu_{1}^{(3)}$ means that the second column $\left[\mu_{1}(x, t ; k)\right]_{2}$ is bounded and analytic in $D_{3}$,
(c) $\mu_{3}^{(12)}$ means that $\left[\mu_{3}(x, t ; k)\right]_{2}$ is bounded and analytic in $D_{1} \cup D_{2}$, etc. 
Let $\left\{\Phi_{j}\right\}_{j=1}^{3}$ be the $2 \times 2$ matrix-valued functions defined in Proposition 1 . Then in their domains of definition the functions $\left\{\Phi_{l}(x, t ; k)\right\}_{l=1}^{3}$ satisfy both equations of the Lax pair, and their determinants (2.2) do not vanish. Hence they are linearly dependent and satisfy the following dependence relations:

$$
\begin{aligned}
& \Phi_{3}(x, t ; k)=\Phi_{2}(x, t ; k) s(k), \quad k \in \mathbb{R}, \\
& \Phi_{1}(x, t ; k)=\Phi_{2}(x, t ; k) S(k), \quad k \in \Sigma,
\end{aligned}
$$

where $s(k)$ and $S(k)$ are defined by

$$
\begin{aligned}
s(k) & :=\Phi_{3}(0,0, k)=:\left(\begin{array}{cc}
\bar{a}(\bar{k}) & b(k) \\
-\bar{b}(\bar{k}) & a(k)
\end{array}\right), \\
S(k) & :=\Phi_{1}(0,0, k)=:\left(\begin{array}{cc}
\bar{A}(\bar{k}) & B(k) \\
-\bar{B}(\bar{k}) & A(k)
\end{array}\right) .
\end{aligned}
$$

Furthermore, the scattering relations (2.7) and (2.8) yield

$$
\Phi_{1}(x, t ; k)=\Phi_{3}(x, t ; k) T(k),
$$

where

$$
T(k)=s^{-1}(k) S(k) .
$$

We denote by $\left\{T_{i j}(k)\right\}_{i, j=1}^{2}$ the entries of the $2 \times 2$ matrix $T(k)$. Then (2.9)-(2.10) imply:

$$
\begin{aligned}
& T_{11}(k)=\bar{T}_{22}(\bar{k})=a(k) \bar{A}(\bar{k})+b(k) \bar{B}(\bar{k}), \\
& T_{12}(k)=-\bar{T}_{21}(\bar{k})=a(k) B(k)-b(k) A(k) .
\end{aligned}
$$

We define

$$
c(k):=\frac{T_{21}(k)}{T_{11}(k)}-\frac{\bar{b}(\bar{k})}{a(k)}=-\frac{\bar{B}(\bar{k})}{a(k) T_{11}(k)},
$$

which is analytic and bounded in $k \in D_{2}$ and is $\mathrm{O}\left(k^{-1}\right)$ as $k \rightarrow \infty$. That follows from the definition of $c(k)$ and from the corresponding properties of the functions $a(k), b(k), A(k)$, $B(k)$ described below and in [6]. Let us also denote

$$
r(k):=\frac{\bar{b}(k)}{a(k)} \text { for } k \in \mathbb{R}
$$

the "reflection coefficient" of the $x$-problem, and

$$
\rho(k):=c(k)+r(k) .
$$

Let $q_{0}(x) \in \mathcal{S}\left(\mathbb{R}_{+}\right)$. Then the map

$$
\mathbb{S}_{x}:\left\{q_{0}(x)\right\} \longmapsto\{a(k), b(k)\}
$$

defined by (2.7), (2.9) has the following properties.

Properties of $\boldsymbol{a}(\boldsymbol{k}), \boldsymbol{b}(\boldsymbol{k})$. The spectral functions $a(k)$ and $b(k)$ satisfy:

(i) $a(k), b(k)$ are analytic and bounded for $k \in \mathbb{C}_{+}$.

(ii) $a(k), b(k) \in C^{\infty}(\mathbb{R})$.

(iii) $|a(k)|^{2}+|b(k)|^{2} \equiv 1, k \in \mathbb{R}$.

(iv) $a(k)=1+\mathrm{O}\left(k^{-1}\right), b(k)=\mathrm{O}\left(k^{-1}\right), k \rightarrow \infty$. 
The map $\mathbb{S}_{x}$ has an inverse

$$
\mathbb{Q}_{x}:\{a(k), b(k)\} \longmapsto q_{0}(x)
$$

given by:

$$
q_{0}(x)=2 \mathrm{i} \lim _{k \rightarrow \infty} k M_{12}^{(x)}(x, k),
$$

where $M^{(x)}(x, k)$ is the unique solution of some Riemann-Hilbert problem $\mathrm{RH}_{x}$ [6].

Now let

$$
\begin{aligned}
& g_{0}(t):=q(0, t)=a \mathrm{e}^{2 \mathrm{i} \omega t} \\
& g_{1}(t):=q_{x}(0, t)=2 \mathrm{i} a b \mathrm{e}^{2 \mathrm{i} \omega t}+v_{1}(t) \text { with } v_{1}(t) \in \mathcal{S}\left(\mathbb{R}_{+}\right) .
\end{aligned}
$$

Then the map

$$
\mathbb{S}_{t}:\left\{g_{0}(t), g_{1}(t)\right\} \longmapsto\{A(k), B(k)\}
$$

defined by $(2.8),(2.10)$ has the following properties.

Properties of $\boldsymbol{A}(\boldsymbol{k}), \boldsymbol{B}(\boldsymbol{k})$. The spectral functions $A(k)$ and $B(k)$ satisfy:

(i) $A(k), B(k)$ are analytic and bounded for $k \in \Omega_{+}=D_{1} \cup D_{3}$.

(ii) $A(k), B(k) \in C^{\infty}(\Sigma \backslash\{E, \bar{E}\}), E=-b+\mathrm{i} a, \bar{E}=-b-\mathrm{i} a$.

(iii) $A(k) \bar{A}(\bar{k})+B(k) \bar{B}(\bar{k}) \equiv 1$ for $k \in \Sigma$.

(iv) $A(k)-\frac{1}{2}\left(\nu(k)+\frac{1}{\nu(k)}\right)$ and $B(k)-\frac{1}{2} \mathrm{e}^{\mathrm{i} \alpha}\left(\nu(k)-\frac{1}{\nu(k)}\right)$ are bounded for $k \in \bar{\Omega}_{+}$.

(v) $A(k)=1+\mathrm{O}\left(k^{-1}\right)$ and $B(k)=\mathrm{O}\left(k^{-1}\right)$ for $k \rightarrow \infty$.

The map $\mathbb{S}_{t}$ has an inverse

$$
\mathbb{Q}_{t}:\{A(k), B(k)\} \longmapsto\left\{g_{0}(t), g_{1}(t)\right\}
$$

given by

$$
\begin{aligned}
& g_{0}(t)=2 \mathrm{i} \lim _{k \rightarrow \infty} k M_{12}^{(t)}(t, k), \\
& g_{1}(t)=\lim _{k \rightarrow \infty}\left[4 k^{2} M_{12}^{(t)}(t, k)+2 \mathrm{i} g_{0}(t) k M_{22}^{(t)}(t, k)\right],
\end{aligned}
$$

where $M^{(t)}(t, k)$ is the unique solution of some Riemann-Hilbert problem $\mathrm{RH}_{t}$ [6].

The spectral functions satisfy the "global relation"

$$
b(k) A(k)-a(k) B(k) \equiv 0 \quad \text { for } k \in D_{1} .
$$

The global relation yields that $T_{12}(k) \equiv 0$ for $k \in D_{1}$ and $T_{21}(k) \equiv 0$ for $k \in D_{4}$. In particular, it means that the spectral term (2.16) vanishes for $k \geq \kappa_{+}$:

$$
\rho(k) \equiv 0 \text { for } k \in\left[\kappa_{+},+\infty\right) .
$$

The function $c(k)$ defined by (2.14) is analytic in $D_{2}$ and has a jump across the contour $\gamma$ :

$$
f(k):=c_{-}(k)-c_{+}(k)=\frac{-\mathrm{ie}^{-\mathrm{i} \alpha}}{T_{11}^{-}(k) T_{11}^{+}(k)} \text { for } k \in \gamma .
$$


The function $\rho(k)$ and all its derivatives have jumps at $k=\kappa_{-}$. Since $r(k)$ is smooth we have for $l=0,1,2, \ldots$

$$
\left.\frac{\mathrm{d}^{l}}{\mathrm{~d} k^{l}} \rho(k)\right|_{k=\kappa_{-}-0}-\left.\frac{\mathrm{d}^{l}}{\mathrm{~d} k^{l}} \rho(k)\right|_{k=\kappa_{-}+0}=f_{l}\left(\kappa_{-}\right):=\left.\frac{\mathrm{d}^{l}}{\mathrm{~d} k^{l}} c(k)\right|_{k=\kappa_{-}-0}-\left.\frac{\mathrm{d}^{l}}{\mathrm{~d} k^{l}} c(k)\right|_{k=\kappa_{-}+0} .
$$

There is also one more general constraint on the $t$-scattering data, the "dispersion relation"

$$
\begin{aligned}
& 2 \operatorname{Re} \int_{\gamma} \log \left(f(k) \delta_{0}(k)\right) \frac{\mathrm{d} k}{X_{+}(k)}=\frac{\pi}{2}-\pi \alpha, \\
& \delta_{0}(k):=\exp \left\{\frac{\mathrm{i}}{\pi} \int_{-\infty}^{\kappa_{+}} \frac{\log \left(1+|\rho(s)|^{2}\right) \mathrm{d} s}{s-k}\right\} .
\end{aligned}
$$

We will explain the appearance of this equation in Remark 2, at the end of Section 5.4.

\section{The Basic Riemann-Hilbert Problem}

The relations among the eigenfunctions (2.7)-(2.13) can be rewritten in the form of a Riemann-Hilbert problem $\mathrm{RH}_{x t}$ :

$$
M_{-}(x, t ; k)=M_{+}(x, t ; k) J(x, t ; k), \quad k \in \Sigma,
$$

which is connected with the IBV problem (1.1). The orientation of the contour

$$
\Sigma=\mathbb{R} \cup \gamma \cup \bar{\gamma} \cup \Gamma \cup \bar{\Gamma}
$$

is shown in Figure 2.

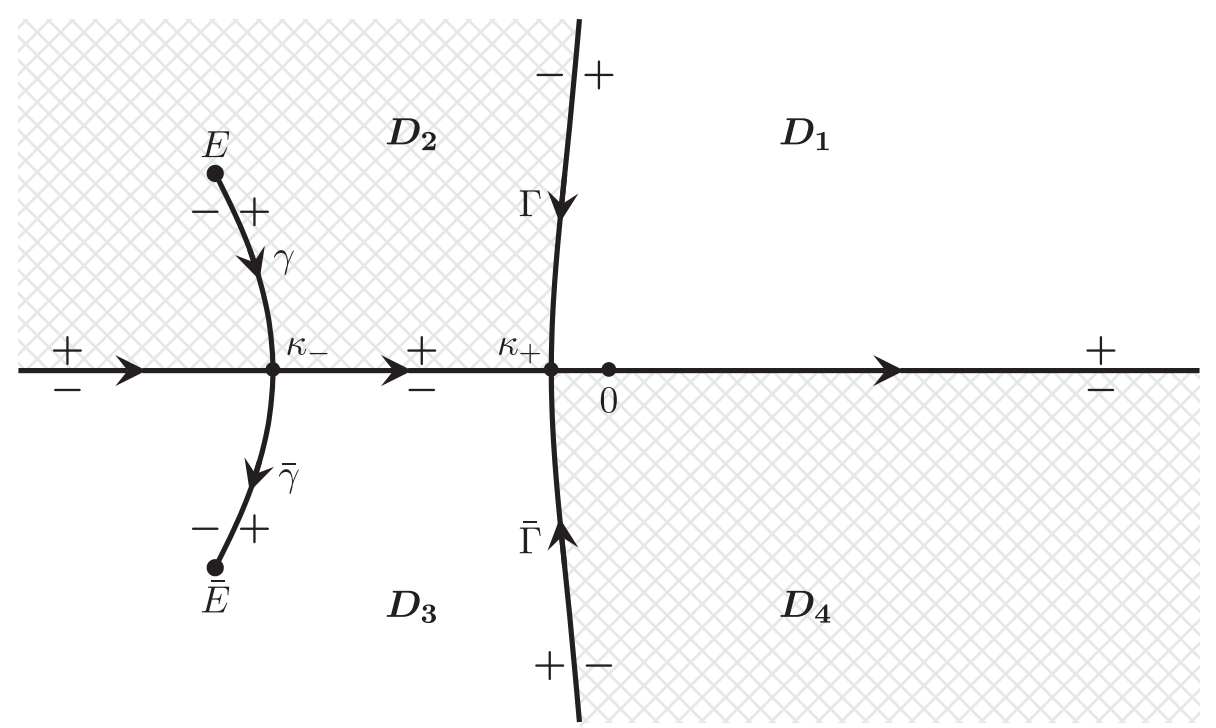

Figure 2. The oriented contour $\Sigma$ for the case $b^{2}>2 a^{2}, a>0, b>0$ 
The boundary values. In (3.1) $M_{+}(x, t ; k)$ denotes the boundary value at $k \in \Sigma$ of the $2 \times 2$ matrix-valued function $M(x, t ; k)$ from the left of the oriented contour $\Sigma$, while $M_{-}(x, t ; k)$ denotes the boundary value from the right, see Figure 2 .

The phase function. We denote

$$
\xi:=\frac{x}{4 t}
$$

Then the "phase function" is defined by

$$
\theta(k, \xi):=2 k^{2}+4 \xi k=2 k^{2}+\frac{k x}{t} .
$$

The jump matrix $\boldsymbol{J}(\boldsymbol{x}, \boldsymbol{t} ; \boldsymbol{k})$. The jump matrix $J(x, t ; k)$ is given by different formulas:

$$
\begin{aligned}
& J(x, t ; k)=\left\{\begin{array}{cc}
\left(\begin{array}{cc}
1 & -\bar{\rho}(k) \mathrm{e}^{-2 \mathrm{i} t \theta(k)} \\
-\rho(k) \mathrm{e}^{2 \mathrm{i} t \theta(k)} & 1+|\rho(k)|^{2}
\end{array}\right), & k \in\left(-\infty, \kappa_{+}\right), \\
\left(\begin{array}{cc}
1 & -\bar{r}(k) \mathrm{e}^{-2 \mathrm{i} t \theta(k)} \\
-r(k) \mathrm{e}^{2 \mathrm{i} t \theta(k)} & 1+|r(k)|^{2}
\end{array}\right), & k \in\left(\kappa_{+}, \infty\right),
\end{array}\right. \\
& J(x, t ; k)= \begin{cases}\left(\begin{array}{cc}
1 & 0 \\
c(k) \mathrm{e}^{2 \mathrm{i} t \theta(k)} & 1
\end{array}\right), & k \in \Gamma, \\
\left(\begin{array}{cc}
1 & \bar{c}(\bar{k}) \mathrm{e}^{-2 \mathrm{i} t \theta(k)} \\
0 & 1
\end{array}\right), & k \in \bar{\Gamma} .\end{cases} \\
& J(x, t ; k)= \begin{cases}\left(\begin{array}{cc}
1 & 0 \\
f(k) \mathrm{e}^{2 \mathrm{i} t \theta(k)} & 1
\end{array}\right), & k \in \gamma, \\
\left(\begin{array}{cc}
1 & -\bar{f}(\bar{k}) \mathrm{e}^{-2 \mathrm{i} t \theta(k)} \\
0 & 1
\end{array}\right), & k \in \bar{\gamma} .\end{cases}
\end{aligned}
$$

Here $c(k), r(k), \rho(k)$ and $f(k)$ are defined by (2.14), (2.15), (2.16) and (2.21), respectively.

In presence of discrete spectrum the following residue conditions hold:

$$
\begin{array}{ll}
\operatorname{res}_{k=k_{j}}[M(x, t ; k)]_{1}=\mathrm{i} m_{j}^{1} \mathrm{e}^{2 \mathrm{i}\left(k_{j} x+2 k_{j}^{2} t\right)}\left[M\left(x, t ; k_{j}\right)\right]_{2}, & k_{j} \in D_{1}, \\
\operatorname{res}_{k=z_{j}}[M(x, t ; k)]_{1}=\mathrm{i} m_{j}^{2} \mathrm{e}^{2 \mathrm{i}\left(z_{j} x+2 z_{j}^{2} t\right)}\left[M\left(x, t ; z_{j}\right)\right]_{2}, & z_{j} \in D_{2}, \\
\operatorname{res}_{k=\bar{z}_{j}}[M(x, t ; k)]_{2}=-\mathrm{i} \bar{m}_{j}^{2} \mathrm{e}^{-2 \mathrm{i}\left(\bar{z}_{j} x+2 \bar{z}_{j}^{2} t\right)}\left[M\left(x, t ; \bar{z}_{j}\right)\right]_{1}, & \bar{z}_{j} \in D_{3}, \\
\operatorname{res}_{k=\bar{k}_{j}}[M(x, t ; k)]_{2}=-\mathrm{i} \bar{m}_{j}^{1} \mathrm{e}^{2 \mathrm{i}\left(\bar{k}_{j} x+2 \bar{k}_{j}^{2} t\right)}\left[M\left(x, t ; \bar{k}_{j}\right)\right]_{1}, & \bar{k}_{j} \in D_{4},
\end{array}
$$

where $k_{j}, j=1,2, \ldots, n$ and $z_{j}, j=1,2, \ldots, m$ are simple zeros of the spectral functions $a(k)$ in $D_{1}$ and of $T_{11}(k)$ in $D_{2}$, respectively. The corresponding residues are as follows:

$m_{j}^{1}=\left(\mathrm{i} b\left(k_{j}\right) \dot{a}\left(k_{j}\right)\right)^{-1}, \quad m_{j}^{2}=-\mathrm{i} \operatorname{res}_{k=z_{j}} c(k), \quad \bar{m}_{j}^{1}=\left(\mathrm{i} \bar{b}\left(\bar{k}_{j}\right) \overline{\dot{a}}\left(\bar{k}_{j}\right)\right)^{-1}, \quad \bar{m}_{j}^{2}=\operatorname{ires}_{k=\bar{z}_{j}} \bar{c}(\bar{k})$. 
Then the solution $q(x, t)$ of the IBV problem (1.1) for the NLS equation is given by

$$
q(x, t)=2 \mathrm{i} \lim _{k \rightarrow \infty}(k M(x, t ; k))_{12} .
$$

4. InVERSE $x t$-SCATtERING PROBlem: RECONSTRUCTION OF the SOlUtion $\boldsymbol{q}(\boldsymbol{x}, \boldsymbol{t})$

In this section we recall results from [6].

The Riemann-Hilbert problem $\mathbf{R H}_{x \boldsymbol{t}}$. Find a $2 \times 2$ matrix-valued function $M(x, t ; k)$ such that

(4.1a) $M(x, t ; k)$ is sectionally meromorphic in $k \in \mathbb{C} \backslash \Sigma$.

(4.1b) Its first column $[M(x, t ; k)]_{1}$ has simple poles at $k_{j} \in D_{1}$ and $z_{j} \in D_{2}$; the second column $[M(x, t ; k)]_{2}$ has simple poles at $\overline{k_{j}} \in D_{4}$ and $\overline{z_{j}} \in D_{3}$. The associated residues satisfy the relations (3.5).

(4.1c) $M(x, t ; k)$ satisfies the jump condition

$$
M_{-}(x, t ; k)=M_{+}(x, t ; k) J(x, t ; k), \quad \text { for } k \in \Sigma
$$

where the jump matrix $J(x, t ; k)$ is defined in terms of the spectral functions by (3.4).

(4.1d) $\operatorname{det} M(x, t ; k) \equiv 1$.

(4.1e) Behavior at $k=\infty$ :

$$
M(x, t ; k)=I+\mathrm{O}\left(k^{-1}\right) .
$$

Theorem $1([6])$. Let $q_{0}(x) \in \mathcal{S}\left(\mathbb{R}_{+}\right)$. Suppose that the functions $g_{0}(t)=a \mathrm{e}^{\mathrm{i} \alpha} \mathrm{e}^{2 \mathrm{i} \omega t}$ and $g_{1}(t)=2 \mathrm{i} a \mathrm{e}^{\mathrm{i} \alpha} b \mathrm{e}^{2 \mathrm{i} \omega t}+v_{1}(t)$ are such that the spectral functions $\{a(k), b(k), A(k), B(k)\}$ satisfy the global relation

$$
b(k) A(k)-a(k) B(k)=0, \quad k \in D_{1} .
$$

Then:

(i) The above Riemann-Hilbert problem $\mathrm{RH}_{x t}$ has a unique solution $M(x, t ; k)$.

(ii) If we define $q(x, t)$ in terms of this solution by

$$
q(x, t)=2 \mathrm{i} \lim _{k \rightarrow \infty}(k M(x, t ; k))_{12},
$$

then

(a) $q(x, t)$ solves the NLS equation (1.1a),

(b) $q(x, t)$ satisfies the initial-boundary conditions:

$$
q(x, 0)=q_{0}(x), \quad q(0, t)=g_{0}(t), \quad q_{x}(0, t)=g_{1}(t) .
$$

5. Long-time asymptotic analysis of the Riemann-Hilbert Problem

Assumptions. In what follows, we assume that the Riemann-Hilbert data, i.e., the functions $\rho(k), r(k), c(k)$ and $f(k)$ satisfy the following additional properties.

\#1 The function $c(k)$ admits analytic continuation across the cut $\gamma \cup \bar{\gamma}$ connecting $E$ and $\bar{E}$ on the second sheet of the Riemann surface of the function $X(k)$.

\#2 The function $f(k)$ admits a Taylor series expansion at $k=E=-b+\mathrm{i} a$ of the form

$$
f(k)=\sum_{j=0}^{\infty} c_{j}(k-E)^{\frac{2 j+1}{2}} .
$$


We also assume for simplicity that

\#3 The discrete spectrum of the problem is empty, i.e.,

- $a(k)$ does not vanish in $D_{1}$,

- $T_{11}(k)=a(k) \bar{A}(\bar{k})+b(k) \bar{B}(\bar{k})$ does not vanish in $D_{2}$.

It means that the set of eigenvalues $\left\{k_{j}\right\}_{j=1}^{n} \cup\left\{z_{j}\right\}_{j=1}^{m}$ is empty.

In the setting of the basic Riemann-Hilbert problem $\mathrm{RH}_{x t}$, Assumption \#1 makes the choice of the contour $\gamma \cup \bar{\gamma}$ itself flexible, with condition (2.22) always satisfied at the point of intersection with the real axes.

In this section, we will show that there exist four different asymptotic formulae which describe the long-time behavior of the solution $q(x, t)$ of the IBV problem in four different regions of the first quarter of the $x t$-plane.

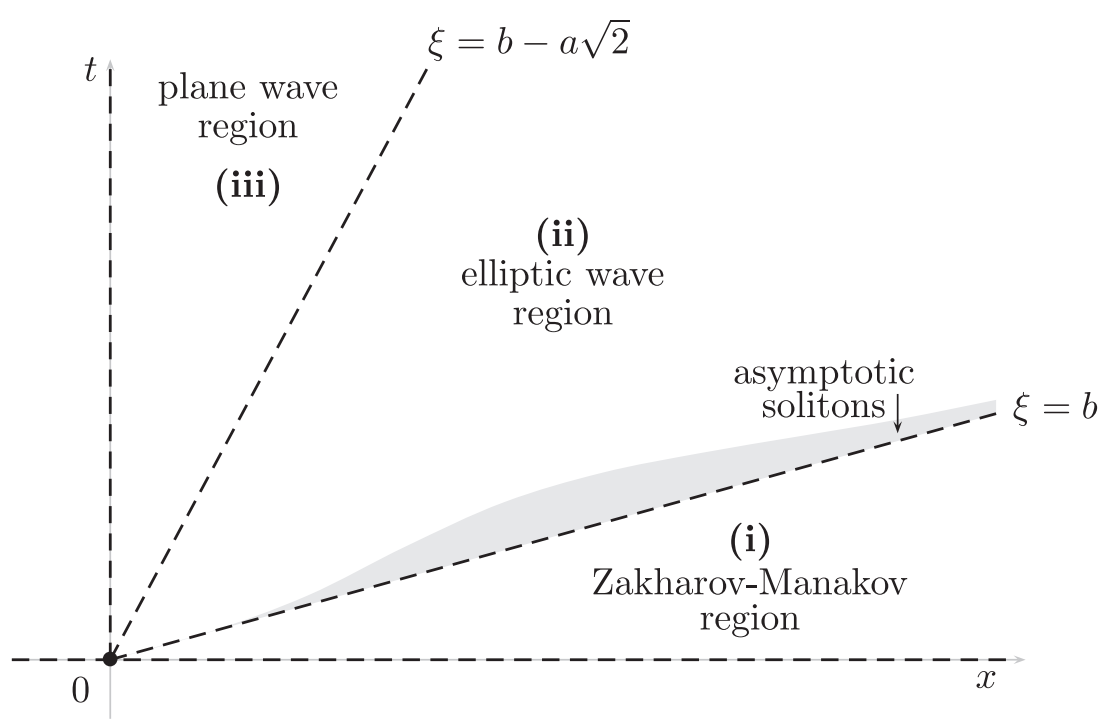

Figure 3. Regions in the $(x, t)$-quarter-plane: $\xi=\frac{x}{4 t}, b=\sqrt{\frac{a^{2}-\omega}{2}}$

For the first region, the so-called Zakharov-Manakov region, we do not actually need the two first extra assumptions just formulated. For the next, the asymptotic solitons region, see Section 5.2, we only need Assumption \#2. For the two remaining regions, Assumption \#1 plays an important technical role in Sections 5.4 and 5.5.

It is worth noticing that if we assume the more general setting (1.2) and take the Riemann-Hilbert data $\rho(k), r(k), c(k)$ and $f(k)$ as the basic functional parameters of our IBV problem, then, of course, we won't have any problem with securing the validity of the assumptions above. The interesting question is how big is the piece of the initial-boundary data which is excluded by the restrictions \#1 and \#2?

5.1. The Zakharov-Manakov region $\boldsymbol{\xi} \equiv \frac{\boldsymbol{x}}{\boldsymbol{4} \boldsymbol{t}}>\boldsymbol{b}$. To study the asymptotic behavior of the Riemann-Hilbert problem $\mathrm{RH}_{x t}$ in the region $x>4 b t$ we use well-known technics from [8]. 
5.1.1. The first transform is as usual:

$$
M(x, t ; k)=M^{(1)}(x, t ; k) \delta^{\sigma_{3}}(k),
$$

where $([14])$

$$
\delta(k)=\exp \left\{\frac{1}{2 \pi \mathrm{i}} \int_{-\infty}^{\kappa_{0}(\xi)} \frac{\log \left(1+|\rho(s)|^{2}\right) \mathrm{d} s}{s-k}\right\}, \quad k \in \mathbb{C} \backslash\left(-\infty, \kappa_{0}(\xi)\right],
$$

and

$$
\kappa_{0}(\xi)=-\xi=-\frac{x}{4 t}
$$

is the stationary point of the phase function

$$
\theta(k, \xi)=2 k^{2}+4 \xi k=2 k^{2}+\frac{k x}{t} .
$$

5.1.2. The next transformation is:

$$
M^{(2)}(x, t ; k)=M^{(1)}(x, t ; k) G(k),
$$

where

$$
\begin{aligned}
& G(k)=\left\{\begin{array}{cl}
\left(\begin{array}{cc}
1 & 0 \\
-\hat{r}(k) \delta^{-2}(k) \mathrm{e}^{2 \mathrm{i} t \theta(k)} & 1
\end{array}\right), & k \in D_{1}, \\
\left(\begin{array}{cc}
1 & \bar{r}(\bar{k}) \delta^{2}(k) \mathrm{e}^{-2 \mathrm{i} t \theta(k)} \\
0 & 1
\end{array}\right), & k \in D_{4}, \\
& \arg \left(k-\kappa_{0}\right) \in(7 \pi / 4,2 \pi),
\end{array}\right. \\
& G(k)=\left\{\begin{array}{cl}
\left(\begin{array}{cc}
1 & 0 \\
\hat{c}(k) \delta^{-2}(k) \mathrm{e}^{2 \mathrm{i} t \theta(k)} & 1
\end{array}\right), & k \in D_{1}, \\
\left(\begin{array}{cc}
1-\overline{\hat{c}}(\bar{k}) \delta^{2}(k) \mathrm{e}^{-2 \mathrm{i} t \theta(k)} \\
0
\end{array}\right), & k \in D_{4}, \\
& \arg \left(k-\kappa_{0}\right) \in(3 \pi / 2,7 \pi / 4),
\end{array}\right. \\
& G(k)=\left\{\begin{array}{cl}
\left(\begin{array}{cc}
1 & 0 \\
-\hat{\rho}(k) \delta^{-2}(k) \mathrm{e}^{2 \mathrm{i} t \theta(k)} & 1
\end{array}\right), & k \in D_{2}, \\
\left(\begin{array}{cc}
1 & \bar{\rho}(\bar{k}) \delta^{2}(k) \mathrm{e}^{-2 \mathrm{i} t \theta(k)} \\
0 & 1
\end{array}\right), & k \in D_{3}, \\
\hline & \arg \left(k-\kappa_{0}\right) \in(7 \pi / 4,2 \pi),
\end{array}\right. \\
& G(k)=\left(\begin{array}{cc}
1 & 0 \\
0 & 1
\end{array}\right), \quad \begin{array}{l}
\left.k \in D_{2}, \pi / 4<\arg \left(k-\kappa_{0}\right)<3 \pi / 4\right), \\
k \in D_{3}, \quad 7 \pi / 4>\arg \left(k-\kappa_{0}\right)>5 \pi / 4,
\end{array}
\end{aligned}
$$

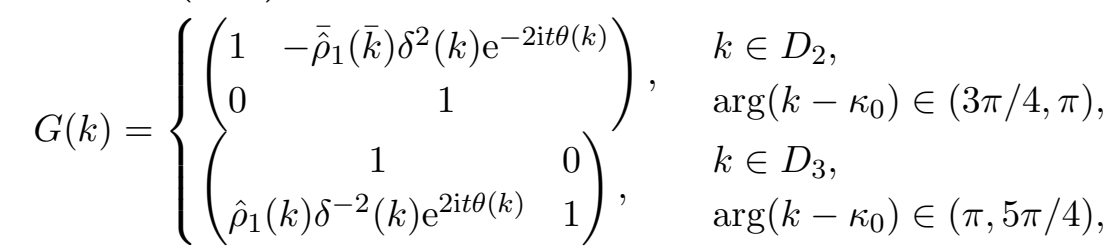

where $\hat{r}(k), \hat{c}(k), \hat{\rho}(k), \hat{\rho}_{1}(k)$ are suitable analytic approximations of the functions $r(k), c(k)$, $\rho(k), \rho_{1}(k)=\rho(k) /\left(1+|\rho(k)|^{2}\right)$ (cf. [11]). Then we obtain the RH problem

$$
M_{-}^{(2)}(x, t ; k)=M_{+}^{(2)}(x, t ; k) J^{(2)}(x, t ; k)
$$




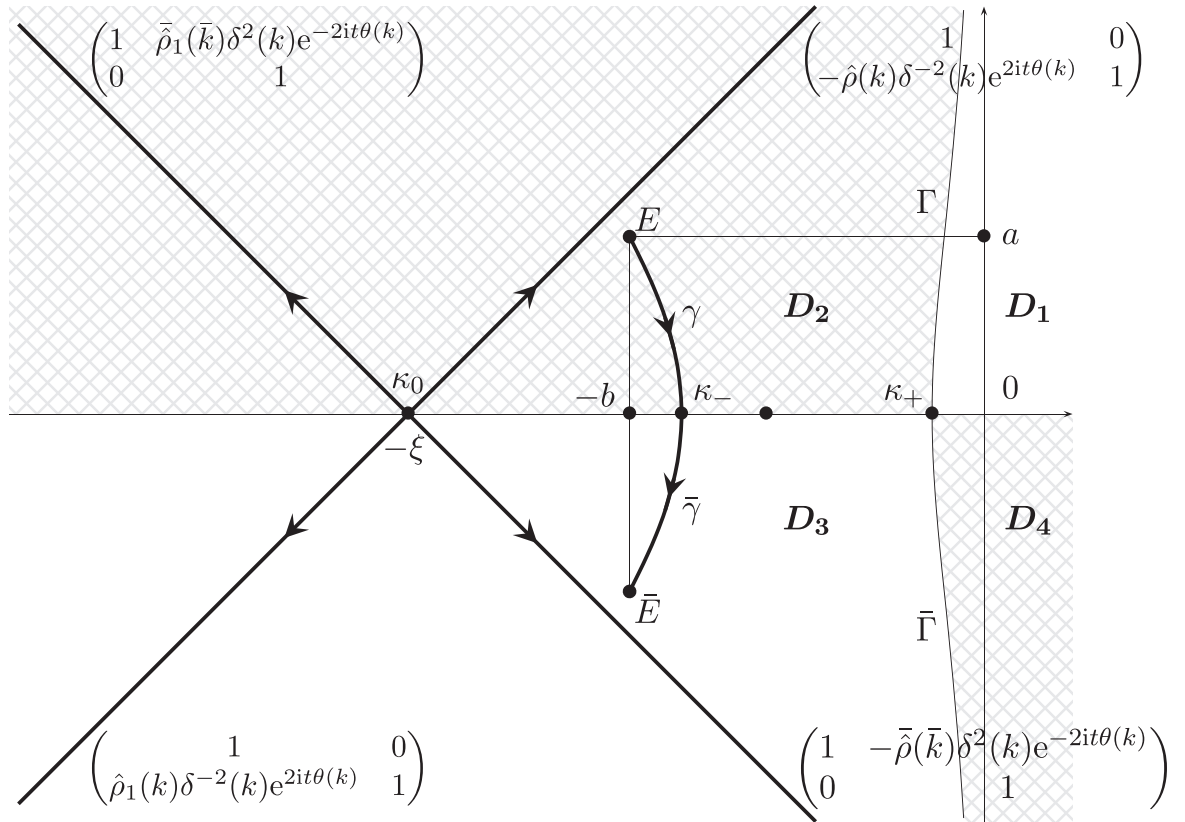

Figure 4 . The contour $\Sigma$ and $J^{(2)}(x, t ; k)$ for $\xi>b$

on the contour $\Sigma=\gamma \cup \bar{\gamma} \cup \operatorname{cross}_{\kappa_{0}}$ depicted in Figure 4. The jump matrices $J^{(2)}(x, t ; k)$ are written for the rays of the cross in Figure 4. Moreover,

$$
J^{(2)}(x, t ; k)=\left\{\begin{array}{cc}
\left(\begin{array}{cc}
1 & 0 \\
\left(\hat{\rho}_{+}(k)-\hat{\rho}_{-}(k)+f(k)\right) \delta^{-2}(k) \mathrm{e}^{2 \mathrm{i} t \theta(k)} & 1
\end{array}\right), & k \in \gamma, \\
\left(\begin{array}{cc}
1 & \left(\overline{\hat{\rho}}_{-}(\bar{k})-\overline{\hat{\rho}}_{+}(\bar{k})-\bar{f}(\bar{k})\right) \delta^{2}(k) \mathrm{e}^{-2 \mathrm{i} t \theta(k)} \\
0 & 1
\end{array}\right), & k \in \bar{\gamma} .
\end{array}\right.
$$

In virtue of the inequality $\xi \equiv \frac{x}{4 t}>b$ and taking into account (2.22) we see that

$$
J^{(2)}(x, t ; k)=I+\mathrm{O}\left(t^{-\infty}\right)
$$

as $t \rightarrow+\infty$, and uniformly in $k \in \gamma \cup \bar{\gamma}$. Hence, in the region $\xi>b$ the jump across the arc $\gamma \cup \bar{\gamma}$ does not contribute to the main term of the asymptotics of the solution.

5.1.3. The final transformation is:

$$
M^{(2)}(x, t ; k)=X(x, t ; k) M^{\text {as }}(x, t ; k),
$$

where the matrix $M^{\text {as }}(x, t ; k)$ solves the standard model problem associated with the stationary phase point $\kappa_{0}(\xi)$ and which is given explicitly in terms of parabolic cylinder functions (see e.g. [8]). The function $X(x, t ; k)$ admits the estimate:

$$
X(x, t ; k)=I+\mathrm{O}\left(\frac{\log ^{2} t}{(1+|k|) t^{1 / 2}}\right) .
$$

Thus we come to the following statement (cf. [14]). 
Theorem 2 (Zakharov-Manakov region, $\xi>b$ ). Suppose that all conditions of Theorem 1 and condition \#3 are satisfied.

Then in the region $\xi>b$ the asymptotics of the solution (4.2) has a quasi-linear dispersive character, i.e., it is described by Zakharov-Manakov type formulas:

$$
\begin{array}{r}
q(x, t)=t^{-1 / 2} \alpha\left(-\frac{x}{4 t}\right) \exp \left\{\frac{\mathrm{i} x^{2}}{4 t}+2 \mathrm{i} \alpha^{2}\left(-\frac{x}{4 t}\right) \log t+\mathrm{i} \phi\left(-\frac{x}{4 t}\right)\right\}+\mathrm{o}\left(t^{-1 / 2}\right), \\
t \rightarrow+\infty, \quad \frac{x}{4 t}>b>0,
\end{array}
$$

with the amplitude $\alpha$ and the phase $\phi$ given by

$$
\begin{aligned}
& \alpha^{2}(k)=\frac{1}{4 \pi} \log \left(1+|\rho(k)|^{2}\right), \\
& \phi(k)=6 \alpha^{2}(k) \log 2+\frac{3 \pi}{4}+\arg \rho(k)+\arg \Gamma\left(-2 \mathrm{i} \alpha^{2}(k)\right)+4 \int_{-\infty}^{k} \log |\mu-k| \mathrm{d} \alpha^{2}(\mu),
\end{aligned}
$$

where $\Gamma(z)$ denotes Euler's gamma-function.

5.2. Asymptotic solitons region $b-\frac{N \log t}{8 a t}<\xi \equiv \frac{x}{4 t}<b$. In what follows we always assume for simplicity condition \#3: the discrete spectrum of the problem is empty, i.e., $a(k)$ and $T_{11}(k)$ do not vanish.

5.2.1. Let us perform the same transforms as in $\S \S 5.1 .1-5.1 .2$ :

$$
M(x, t ; k) \rightsquigarrow M^{(1)}(x, t ; k) \rightsquigarrow M^{(2)}(x, t ; k) .
$$

In this case the matrix Riemann-Hilbert problem is as follows:

$$
M_{-}^{(2)}(x, t ; k)=M_{+}^{(2)}(x, t ; k) J^{(2)}(x, t ; k)
$$

with contour $\Sigma=\gamma \cup \bar{\gamma} \cup \operatorname{cross}_{\kappa_{0}}$, see Fig. 5. For the jump matrix $J^{(2)}(x, t ; k)$ for $k \in \operatorname{cross}_{\kappa_{0}}$, see Fig. 5. For $k \in \gamma \cup \bar{\gamma}$ :

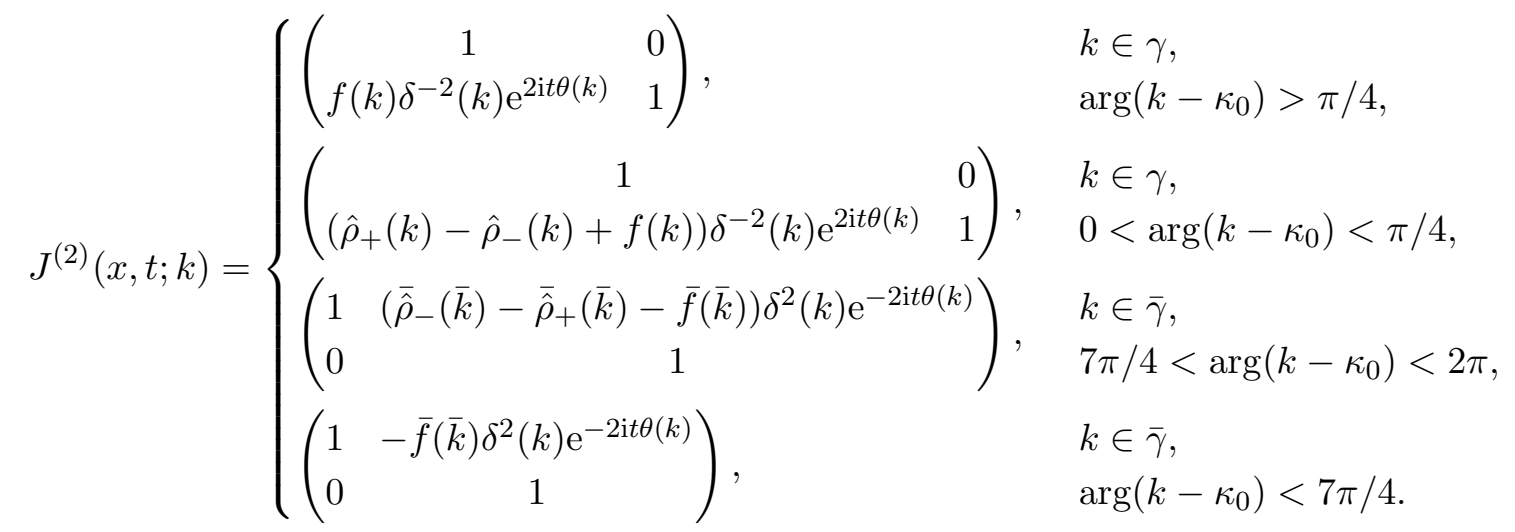




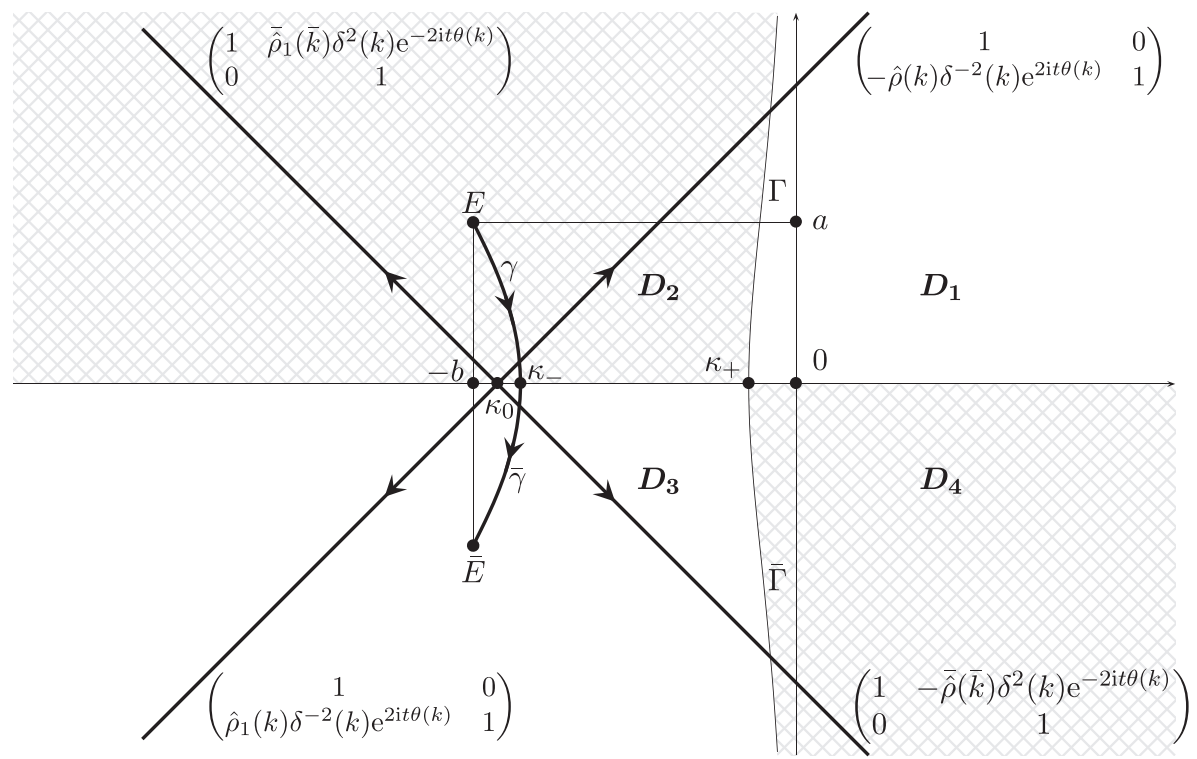

Figure 5. The contour $\Sigma$ and $J^{(2)}(x, t ; k)$ for $b-\frac{N}{8 a} \frac{\log t}{t}<\xi<b$

5.2.2. The next transformation is:

$$
M^{(2)}(x, t ; k)=\hat{\Phi}(x, t ; k) T_{f}(x, t ; k),
$$

where

$$
T_{f}(x, t ; k)= \begin{cases}\left(\begin{array}{cc}
1 & 0 \\
K_{f}(x, t ; k) & 1
\end{array}\right), & \text { for }|k-E|<\varepsilon \\
\left(\begin{array}{cc}
1 & \bar{K}_{f}(x, t ; \bar{k}) \\
0 & 1
\end{array}\right), & \text { for }|k-\bar{E}|<\varepsilon \\
I, & \text { otherwise }\end{cases}
$$

with

$$
\begin{aligned}
& K_{f}(x, t ; k)=\frac{1}{2 \pi \mathrm{i}} \int_{\gamma \cap|k-E|<\varepsilon} \frac{f(s) \delta^{-2}(s) \mathrm{e}^{2 \mathrm{i} t \theta(s)}}{s-k} \mathrm{~d} s, \\
& \bar{K}_{f}(x, t ; \bar{k})=-\frac{1}{2 \pi \mathrm{i}} \int_{\bar{\gamma} \cap|k-\bar{E}|<\varepsilon} \frac{\bar{f}(\bar{s}) \delta^{2}(s) \mathrm{e}^{-2 \mathrm{i} t \theta(s)}}{s-k} \mathrm{~d} s .
\end{aligned}
$$

Then the Riemann-Hilbert problem takes the form:

$$
\hat{\Phi}_{-}(x, t ; k)=\hat{\Phi}_{+}(x, t ; k) J_{\hat{\Phi}}(x, t ; k), \quad k \in \Sigma^{\prime}
$$

on the contour $\Sigma^{\prime}=\gamma_{\varepsilon} \cup \bar{\gamma}_{\varepsilon} \cup C_{\varepsilon} \cup \bar{C}_{\varepsilon} \cup \operatorname{cross}_{\kappa_{0}}$ where

$$
\begin{aligned}
& \gamma_{\varepsilon}=\{k \in \gamma|| k-E \mid \geq \varepsilon\}, \\
& C_{\varepsilon}=\{k|| k-E \mid=\varepsilon\} .
\end{aligned}
$$


See Figure 6.

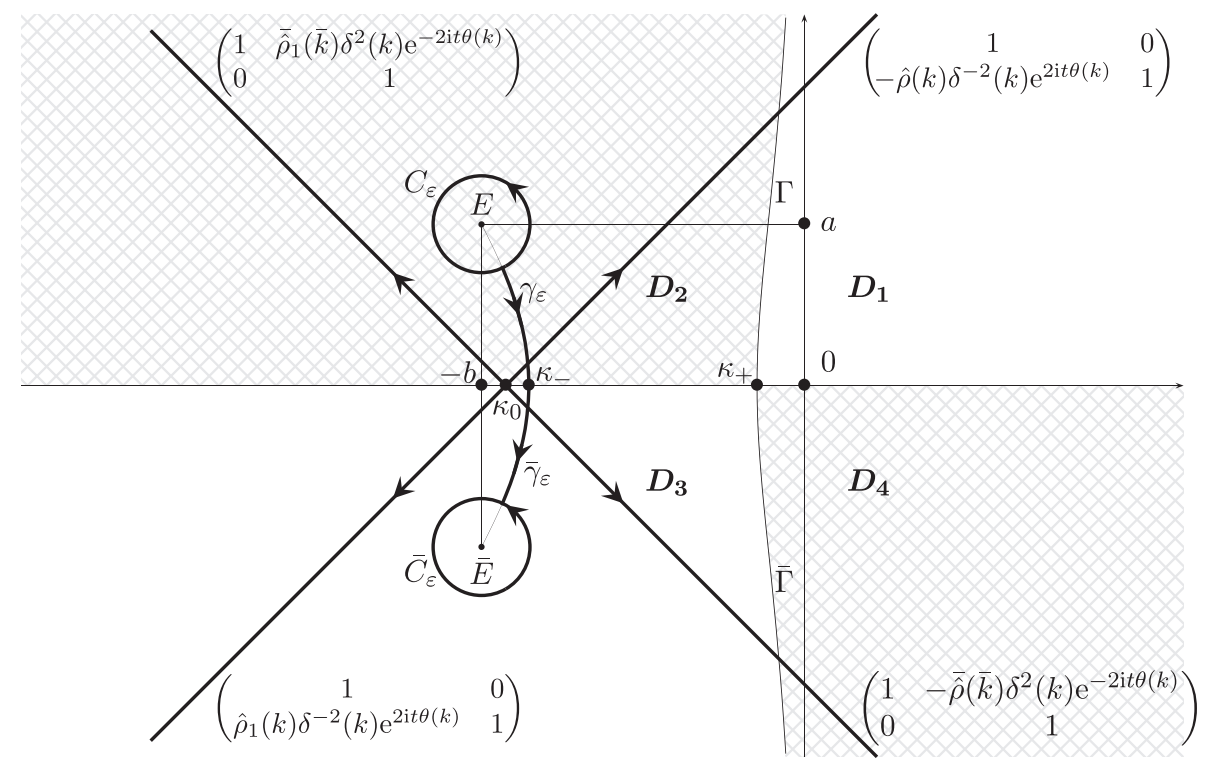

Figure 6. The contour $\Sigma^{\prime}$ and $J_{\hat{\Phi}}(x, t ; k)$ for $b-\frac{N}{8 a} \frac{\log t}{t}<\xi<b$

The jump matrix is

$$
J_{\hat{\Phi}}(x, t ; k)= \begin{cases}J^{(2)}(x, t ; k) & \text { for } k \in \gamma_{\varepsilon} \cup \bar{\gamma}_{\varepsilon} \cup \operatorname{cross}_{\kappa_{0}}, \\ T_{f}(x, t ; k) & \text { for } k \in C_{\varepsilon} \cup \bar{C}_{\varepsilon} .\end{cases}
$$

Due to assumption \#2 on the behavior of $f(k)$ near $k=E$, the functions $K_{f}(x, t ; k)$ and $\bar{K}_{f}(x, t ; \bar{k})$ are of the form:

$$
\begin{aligned}
& K_{f}(x, t ; k)=F_{N}(k, t, \xi)+R(k, t, \xi), \\
& \bar{K}_{f}(x, t ; \bar{k})=\bar{F}_{N}(\bar{k}, t, \xi)+\bar{R}(\bar{k}, t, \xi),
\end{aligned}
$$

where

$$
F_{N}(k, t, \xi)=\sum_{j=0}^{N} \frac{d_{j}(t, \xi)}{t^{j+3 / 2}} \frac{\mathrm{e}^{2 \mathrm{i} t \theta(E)}}{(k-E)^{j+1}}
$$

In the region

$$
b-\frac{N \log t}{8 a t}<\xi=\frac{x}{4 t}<b
$$

we have, for $t \rightarrow+\infty$ :

$$
\begin{aligned}
& \mathrm{e}^{2 \mathrm{i} t \theta(E)}=\mathrm{O}\left(t^{N}\right) \\
& d_{j}(t, \xi)=d_{j}+\mathrm{O}\left(t^{-1}\right), \text { where } d_{j}=\text { const } \neq 0 \\
& R(k, t, \xi)=\mathrm{O}\left(t^{-3 / 2}\right)
\end{aligned}
$$


Hence, for $k \in C_{\varepsilon} \cup \bar{C}_{\varepsilon}$ the jump matrix $J_{\hat{\Phi}}(x, t ; k)$ can be written in the form:

$$
J_{\hat{\Phi}}(x, t ; k)=T_{f}(x, t ; k)=T_{f}^{N}(k, t, \xi)+T_{f}^{\mathrm{reg}}(k, t, \xi),
$$

where

$$
\begin{aligned}
& T_{f}^{N}(k, t, \xi)= \begin{cases}\left(\begin{array}{cc}
1 & 0 \\
F_{N}(k, t, \xi) & 1
\end{array}\right), & \text { for }|k-E|=\varepsilon, \\
\left(\begin{array}{cc}
1 & -\bar{F}_{N}(\bar{k}, t, \xi) \\
0 & 1
\end{array}\right), & \text { for }|k-\bar{E}|=\varepsilon,\end{cases} \\
& T_{f}^{\mathrm{reg}}(k, t, \xi)= \begin{cases}\left(\begin{array}{cc}
1 & 0 \\
R(k, t, \xi) & 1
\end{array}\right), & \text { for }|k-E|=\varepsilon, \\
\left(\begin{array}{cc}
1 & -\bar{R}(\bar{k}, t, \xi) \\
0 & 1
\end{array}\right), & \text { for }|k-\bar{E}|=\varepsilon .\end{cases}
\end{aligned}
$$

Let $\Phi^{\mathrm{reg}}(k, t, \xi)$ denote the solution of the "regular" $\mathrm{RH}$ problem

$$
\Phi_{-}^{\mathrm{reg}}(k, t, \xi)=\Phi_{+}^{\mathrm{reg}}(k, t, \xi) J_{\Phi^{\mathrm{reg}}}, \quad k \in \Sigma^{\prime} .
$$

The contour $\Sigma^{\prime}$ is the same as before. The jump matrix $J_{\Phi^{\text {reg }}}$ is obtained from $J_{\hat{\Phi}}$ by the replacement $T_{f}(k, t, \xi) \rightsquigarrow T_{f}^{\mathrm{reg}}(k, t, \xi)$. It is clear that

$$
\Phi^{\mathrm{reg}}(k, t, \xi)=\left[I+\mathrm{O}\left(\frac{\log ^{2} t}{t^{1-2 \varepsilon}}\right)\right] \Phi^{\mathrm{as}}(k, t, \xi), \quad 0<\varepsilon<1 / 2,
$$

where $\Phi^{\text {as }}(k, t, \xi)$ is the same parabolic cylinder model matrix function as above in §5.1.3.

5.2.3. Now we put

$$
\hat{\Phi}(k, t, \xi)=\Phi^{\mathrm{sol}}(k, t, \xi) \Phi^{\mathrm{reg}}(k, t, \xi) .
$$

Soliton model RH-problem. The matrix-valued function $\Phi^{\mathrm{sol}}(k, t, \xi)$ solves the following model RH problem:

- $\Phi^{\mathrm{sol}}(k, t, \xi)$ is analytic in $k \in \mathbb{C} \backslash\left\{C_{\varepsilon} \cup \bar{C}_{\varepsilon}\right\}$.

- $\Phi_{-}^{\mathrm{sol}}(k, t, \xi)=\Phi_{+}^{\mathrm{sol}}(k, t, \xi) J_{\Phi^{\mathrm{sol}}}(k, t, \xi)$ for $k \in C_{\varepsilon} \cup \bar{C}_{\varepsilon}$.

- $\Phi^{\mathrm{sol}}(k, t, \xi)=I+\mathrm{O}\left(k^{-1}\right)$, as $k \rightarrow \infty$.

The contour $C_{\varepsilon} \cup \bar{C}_{\varepsilon}$ is the union of two circles of small radius $\varepsilon>0$ centered at $E$ and $\bar{E}$, respectively. The jump matrix has the form

$$
J_{\Phi_{-}^{\text {sol }}}(k, t, \xi)=\Phi_{+}^{\mathrm{reg}}(k, t, \xi) T_{f}^{N}(k, t, \xi)\left(\Phi_{+}^{\mathrm{reg}}(k, t, \xi)\right)^{-1} .
$$

This problem can be solved purely algebraically. Finally we obtain:

$$
\hat{\Phi}(k, t, \xi)=\Phi^{\mathrm{sol}}(k, t, \xi)\left[I+\mathrm{O}\left(\frac{1}{(1+|k|) t^{1 / 2}}\right)\right], \quad t \rightarrow+\infty,
$$

that yields

$$
q(x, t)=q_{\mathrm{sol}}(x, t)+\mathrm{O}\left(t^{-1 / 2}\right), \quad t \rightarrow+\infty
$$


for the solution of the IBV problem (1.1) in the region $4 b t-\frac{N}{2 a} \log t<x \leq 4 b t$. The explicit formula for an asymptotic soliton chain $q_{\text {sol }}(x, t)$ can be deduced algebraically from the above Riemann-Hilbert problem.

Alternatively, by using the Marchenko approach, this asymptotics was studied in [5], where the following theorem was obtained:

Theorem 3 (asymptotic solitons, [5]). Suppose that all conditions of Theorem 1 and conditions \#2, \#3 are satisfied. Let $N$ be a positive integer.

Then in the region $4 b t-\frac{N}{2 a} \log t<x \leq 4 b t$ the solution (4.2) is an asymptotic soliton chain:

$$
|q(x, t)|^{2}=\sum_{j=1}^{\left[\frac{N+1}{2}\right]} \frac{4 a^{2}}{\cosh ^{2}\left[2 a\left(x-4 b t-x_{j}\right)+\log t^{2 j-1 / 2}\right]}+\mathrm{o}(1)
$$

where

$$
x_{j}=x_{j}^{(0)}-\frac{1}{2 \pi} \int_{-\infty}^{-b} \frac{\log \left[1+|\rho(\lambda)|^{2}\right]}{(\lambda+b)^{2}+a^{2}} \mathrm{~d} \lambda
$$

and the numbers $x_{j}^{(0)}$ depend on $\gamma_{0}^{+}$and $c(k)$.

The first asymptotic soliton $(N=1)$ takes the form:

$$
q_{\mathrm{sol}}(x, t)=-\frac{2 a \mathrm{e}^{2 \mathrm{i} b x+4 \mathrm{i}\left(a^{2}-b^{2}\right) t+\mathrm{i} \varphi_{1}}}{\cosh \left[2 a\left(x-4 b t+\frac{1}{2 a} \log t^{3 / 2}-x_{1}\right)\right]}
$$

where

$$
\varphi_{1}=\arg z_{0}-\frac{1}{\pi} \operatorname{Re} \int_{-\infty}^{\kappa_{0}} \log \left[1+|\rho(s)|^{2}\right] \frac{\mathrm{d} s}{s+b-\mathrm{i} a} .
$$

Here $z_{0}$ depends on $E=-b+\mathrm{i} a$.

5.3. The sector $\mathbf{0} \leq \boldsymbol{\xi} \equiv \frac{\boldsymbol{x}}{\boldsymbol{4} \boldsymbol{t}}<\boldsymbol{b}$. For $0 \leq \xi<b, \operatorname{Im} \theta(k)$ is negative along a part or all of the contour $\gamma \cup \bar{\gamma}$. Therefore the method used in the initial region $(\xi>b)$ will not work. For $0 \leq \xi<b$, we have to follow a modification of the nonlinear steepest descent method as suggested in [10]. Instead of $\theta(k)=2 k^{2}+4 \xi k$ we should find a new phase function $g(k)=g(k, \xi)$, which transforms the original Riemann-Hilbert problem to a model $\mathrm{RH}$ problem of finite-gap type (see [9]). Such a $g$-function does really exist. It leads to a genus zero finite-gap model problem for $0 \leq \xi<b-a \sqrt{2}$ and to a genus one finite-gap model problem for $b-a \sqrt{2}<\xi<b$. Both are explicitly solved, using elementary functions in the first region, $\S 5.4$, and elliptic theta functions in the second region, $\S 5.5$, respectively.

\subsection{Plane wave region $0 \leq \xi \equiv \frac{x}{4 t}<b-a \sqrt{2}$.}

5.4.1. The $g$-function. In the region $0 \leq \xi<b-a \sqrt{2}$ we take as $g$-function (cf. (1.8d)):

$$
g(k)=g(k, \xi)=2(k-b+2 \xi) X(k)=\Omega(k)+4 \xi X(k),
$$

where $X(k)=\sqrt{(k-E)(k-\bar{E})}=\sqrt{(k+b)^{2}+a^{2}}$. This function has the same asymptotic behavior for large $k$ as the initial phase function $\theta(k)$, i.e.

$$
\begin{array}{ll}
g(k)=2 k^{2}+4 \xi k+g_{\infty}(\xi)+\mathrm{O}\left(k^{-1}\right), & k \rightarrow \infty, \text { with } \\
g_{\infty}(\xi)=a^{2}-2 b^{2}+4 b \xi=\omega+4 b \xi, & 0 \leq \xi<b-a \sqrt{2} .
\end{array}
$$




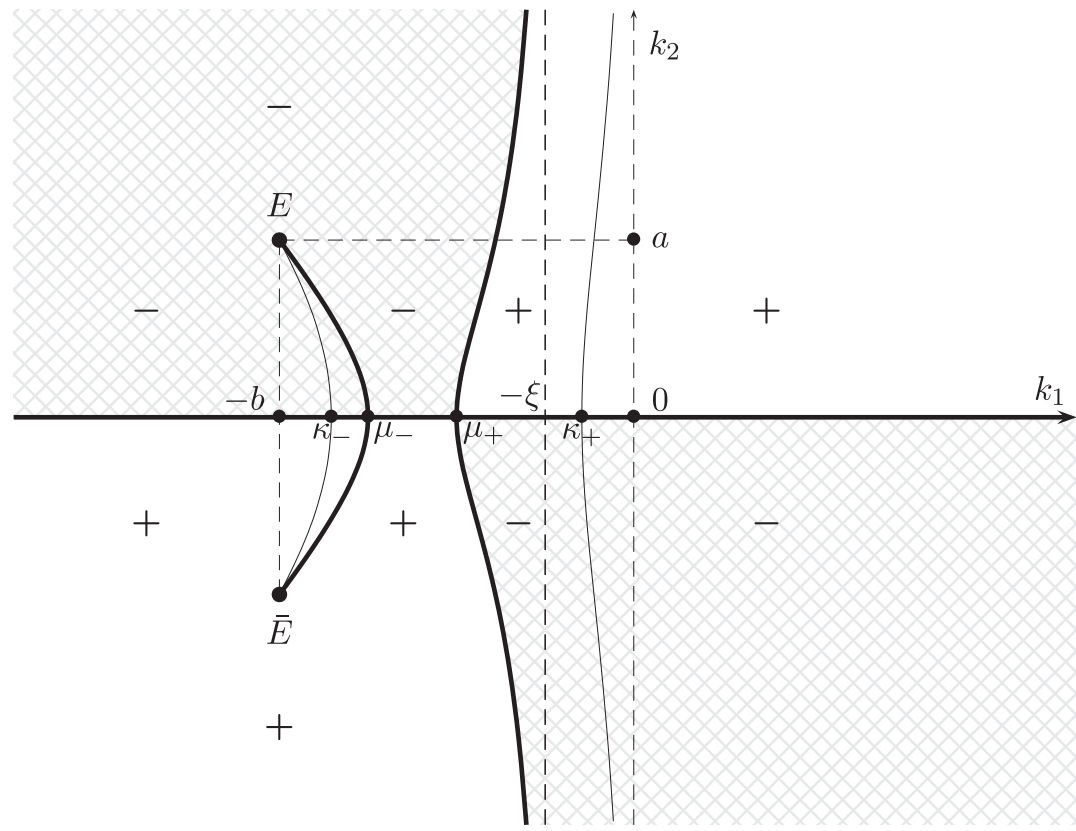

FiguRE 7. The signature table of $\operatorname{Im} g(k)$ for $0<\xi<b-a \sqrt{2}$

The zeros $\mu_{ \pm}$of the differential

$$
\begin{aligned}
\mathrm{d} g(k) & =4 \frac{\left(k-\mu_{-}\right)\left(k-\mu_{+}\right)}{X(k)} \mathrm{d} k \\
& =\mathrm{d}(\Omega(k)+4 \xi X(k)) \\
& =4 \frac{k^{2}+(b+\xi) k+a^{2} / 2+b \xi}{X(k)} \mathrm{d} k
\end{aligned}
$$

are as follows for $0 \leq \xi \leq b-a \sqrt{2}$ :

$$
\mu_{ \pm}(\xi)=-\frac{b+\xi}{2} \pm \sqrt{\frac{(b-\xi)^{2}}{4}-\frac{a^{2}}{2}} .
$$

They are real while $0 \leq \xi \leq \xi_{0}=b-a \sqrt{2}$ and complex conjugate if $\xi>\xi_{0}$. We will see in the next section that for $\xi>\xi_{0}$ the $g$-function should be chosen differently.

In what follows the signature table of the function $\operatorname{Im} g(k)$ for different values of $\xi$ plays a very important role. The lines of separation between the different domains are the real axis

$$
k_{2}=0
$$

and the algebraic curve

$$
k_{2}^{2}=\left(k_{1}^{2}+(b+\xi) k_{1}+\frac{a^{2}}{2}+b \xi\right) \frac{k_{1}-b+2 \xi}{k_{1}+\xi} .
$$

They are indeed given by $\operatorname{Im} g(k)=0$. The signature table of the function $\operatorname{Im} g(k)$ is depicted in Figure 7 for $0 \leq \xi<\xi_{0}$ and in Figure 10 for $\xi=\xi_{0}$. 
5.4.2. We shall now take advantage of condition \#1 imposed on the Riemann-Hilbert data $c(k)$. We deform the contour $\gamma \cup \bar{\gamma}$ to the contour $\gamma_{g} \cup \bar{\gamma}_{g}$ where $\operatorname{Im} g(k)=0$. This contour depends on $\xi$ because it connects the points $E, \mu_{-}(\xi)$, and $\bar{E}$. All functions, that had jumps across $\gamma \cup \bar{\gamma}$, have now jumps across $\gamma_{g} \cup \bar{\gamma}_{g}$ with the same jump relations as they had before the deformation. The basic Riemann-Hilbert problem $\mathrm{RH}_{x t}$ have to be considered now on a new contour: $\Sigma=\mathbb{R} \cup \gamma_{g} \cup \bar{\gamma}_{g} \cup \Gamma \cup \bar{\Gamma}$ and with the new phase function. More precisely, we put

$$
M(x, t ; k)=\mathrm{e}^{\mathrm{i} t g_{\infty}(\xi) \sigma_{3}} M^{(1)}(x, t ; k) \mathrm{e}^{\mathrm{i}\left[k x+2 k^{2} t-t g(k)\right] \sigma_{3}},
$$

where the phase function $g(k)=g(k, \xi)$ is defined in (5.8). Then the matrix $M^{(1)}(x, t ; k)$ satisfies the following $\mathrm{RH}$ problem:

$$
M_{-}^{(1)}(x, t ; k)=M_{+}^{(1)}(x, t ; k) J^{(1)}(x, t ; k)
$$

with the jump matrix

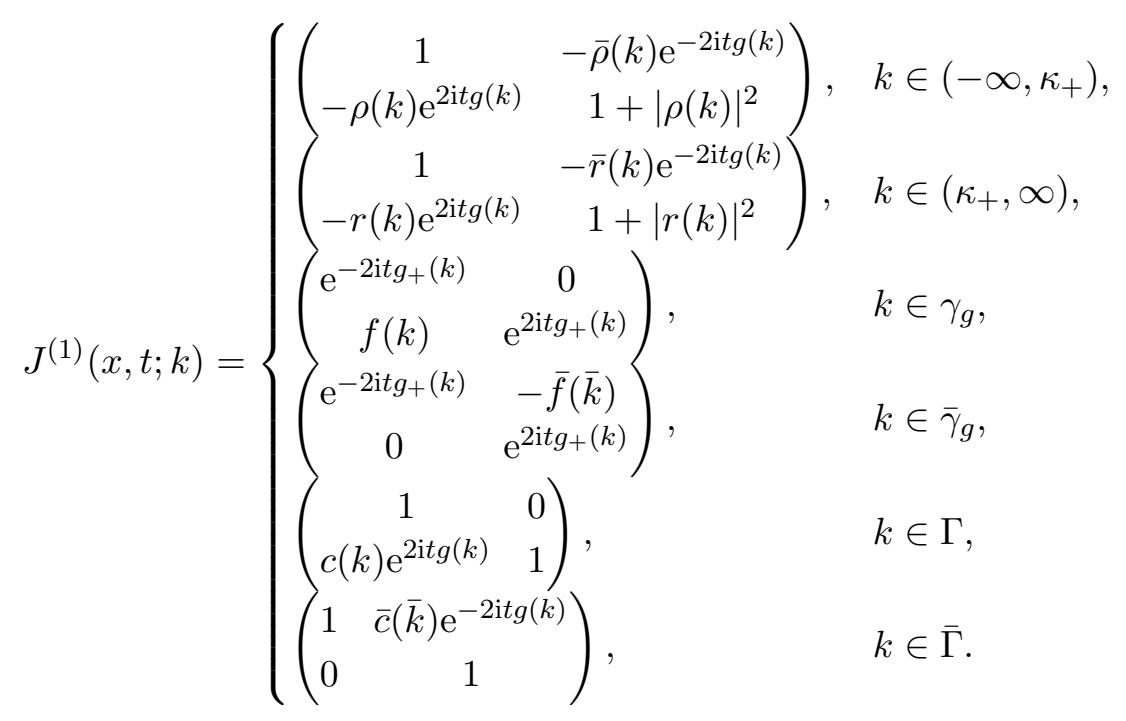

5.4.3. Let us perform the same transformation as before, $\S 5.1 .1$ :

$$
M^{(1)}(x, t ; k)=M^{(2)}(x, t ; k) \delta^{\sigma_{3}}(k) .
$$

The function $\delta(k)$ is defined as in (5.2), but now $\kappa_{0}=\mu_{+}(\xi)$, where $\mu_{+}(\xi)$ is the stationary point of the new phase function $g(k)$. The corresponding jump matrix $J^{(2)}(x, t ; k)$ is factorized as follows:

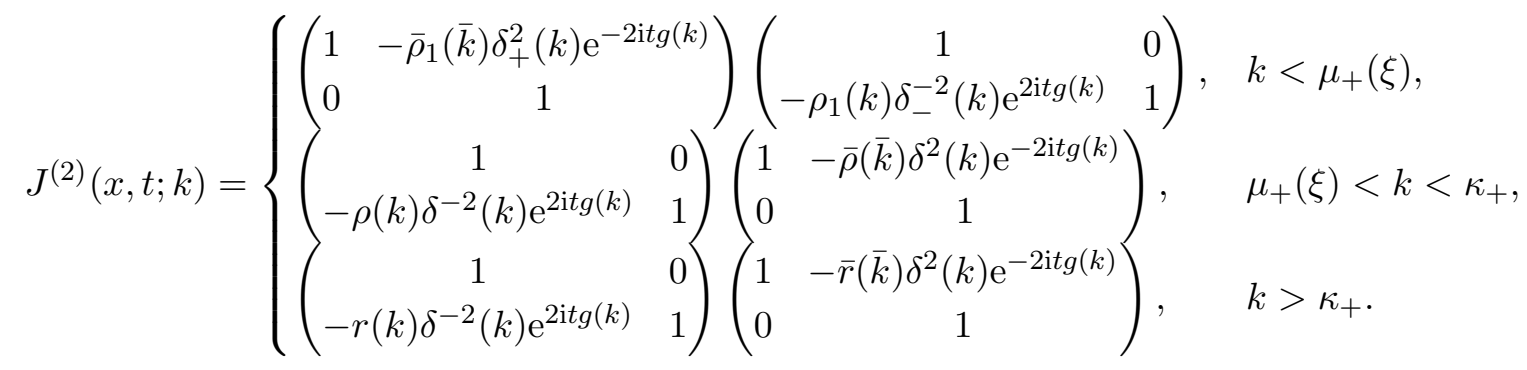


For the remaining $\operatorname{arcs}$ of $\Sigma$ the jump matrix is given by:

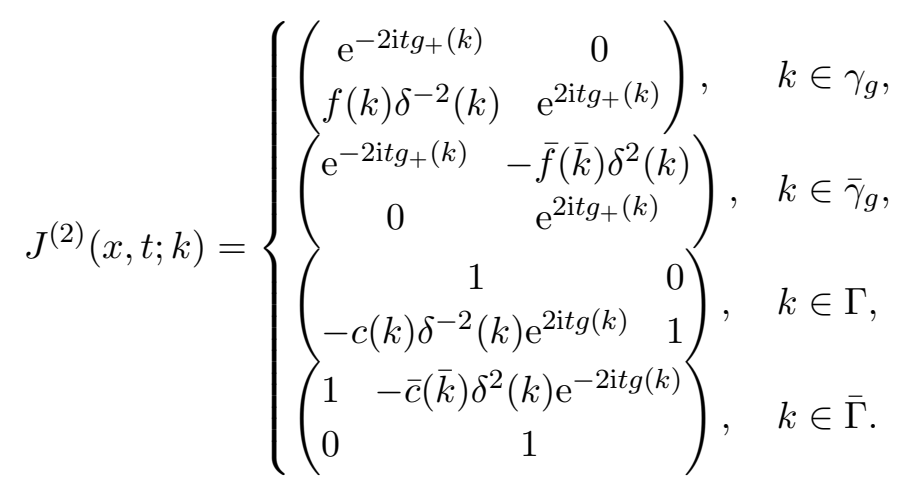

5.4.4. Now we use the same transformation as in $\S 5.1 .2$ :

$$
M^{(3)}(x, t ; k)=M^{(2)}(x, t ; k) G(k),
$$

where $G(k)$ is given by (5.4) with $\theta(k)$ replaced by $g(k)$ and $\kappa_{0}$ by $\mu_{+}(\xi)$. After this $G$ transformation, the Riemann-Hilbert problem becomes:

$$
M_{-}^{(3)}(x, t ; k)=M_{+}^{(3)}(x, t ; k) J^{(3)}(x, t ; k) .
$$

The contour $\Sigma^{(3)}=\gamma_{g} \cup \bar{\gamma}_{g} \cup \operatorname{cross}_{\mu_{+}}$of this RH problem is depicted in Figure 8 .

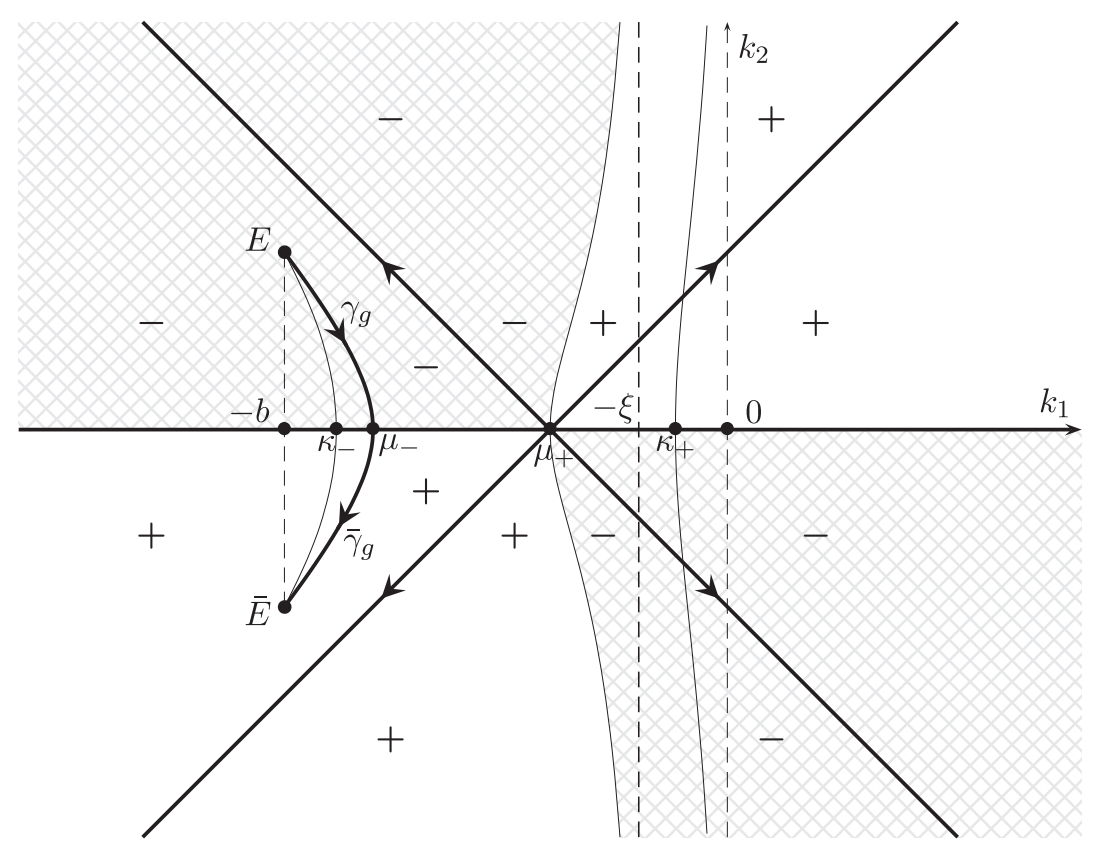

Figure 8. The contour $\Sigma^{(3)}$ of the RH problem for $0<\xi<b-a \sqrt{2}$ 
Let $\Sigma_{\text {cross }}^{(3)}=\left\{k \mid \arg \left(k-\mu_{+}\right)=\frac{\pi}{4}, \frac{3 \pi}{4}, \frac{5 \pi}{4}, \frac{7 \pi}{4}\right\}$. Let $D_{\mu_{+}}$be a small disk centered at $\mu_{+}$. For $k \in \Sigma_{\text {cross }}^{(3)} \backslash\left(D_{\mu_{+}} \cap \Sigma_{\text {cross }}^{(3)}\right)$, the jump matrix admits the following estimate:

$$
J^{(3)}(x, t ; k)=I+\mathrm{O}\left(\mathrm{e}^{-\varepsilon t}\right), \quad \varepsilon>0, \quad t \rightarrow+\infty .
$$

Therefore the main attention we have to pay is to $\gamma_{g} \cup \bar{\gamma}_{g}$, where the jump matrix

$$
J^{(3)}(x, t ; k):=G_{+}^{-1}(k) J^{(2)}(x, t ; k) G_{-}(k)
$$

factorizes as follows:

$$
J^{(3)}= \begin{cases}\left(\begin{array}{cc}
1 & \overline{\hat{\rho}}_{1+}(\bar{k}) \delta^{2}(k) \mathrm{e}^{-2 \mathrm{i} t g_{+}(k)} \\
0 & 1
\end{array}\right) J^{(2)}(x, t ; k)\left(\begin{array}{cc}
1 & -\overline{\hat{\rho}}_{1-}(\bar{k}) \delta^{2}(k) \mathrm{e}^{2 \mathrm{i} t g_{+}(k)} \\
0 & 1
\end{array}\right), & k \in \gamma_{g}, \\
\left(\begin{array}{cc}
1 & 0 \\
-\hat{\rho}_{1+}(k) \delta^{-2}(k) \mathrm{e}^{2 \mathrm{it} g_{+}(k)} & 1
\end{array}\right) J^{(2)}(x, t ; k)\left(\begin{array}{cc}
1 & 0 \\
\hat{\rho}_{1-}(k) \delta^{-2}(k) \mathrm{e}^{-2 \mathrm{i} t g_{+}(k)} & 1
\end{array}\right), & k \in \bar{\gamma}_{g} .\end{cases}
$$

Let $F(k), k \notin \gamma_{g} \cup \bar{\gamma}_{g}$ be a function such that

$$
F_{-}(k) F_{+}(k)=-\mathrm{ie}^{\mathrm{i} \alpha} f(k) \delta^{-2}(k) \text { for } k \in \gamma_{g} .
$$

Here $\alpha$ is the same as in (1.1). Then for $k \in \gamma_{g}$ we can factorize $J^{(2)}(x, t ; k)$ as follows:

$$
\begin{aligned}
& J^{(2)}(x, t ; k):=\left(\begin{array}{cc}
\mathrm{e}^{-2 \mathrm{i} t g_{+}(k)} & 0 \\
f(k) \delta^{-2}(k) & \mathrm{e}^{2 \mathrm{i} t g_{+}(k)}
\end{array}\right) \\
& =\left(\begin{array}{cc}
F_{+}^{-1}(k) & 0 \\
0 & F_{+}(k)
\end{array}\right)\left(\begin{array}{cc}
F_{+}(k) F_{-}^{-1}(k) \mathrm{e}^{-2 \mathrm{i} t g_{+}(k)} & 0 \\
\mathrm{ie}-\mathrm{i} \alpha & F_{-}(k) F_{+}^{-1}(k) \mathrm{e}^{2 \mathrm{i} t g_{+}(k)}
\end{array}\right)\left(\begin{array}{cc}
F_{-}(k) & 0 \\
0 & F_{-}^{-1}(k)
\end{array}\right) \\
& =F_{+}^{-\sigma_{3}}(k)\left(\begin{array}{cc}
1 & -\mathrm{ie}^{\mathrm{i} \alpha} \psi(k) \mathrm{e}^{-2 \mathrm{i} t g_{+}(k)} \\
0 & 1
\end{array}\right)\left(\begin{array}{cc}
0 & \mathrm{ie}^{\mathrm{i} \alpha} \\
\mathrm{ie}^{-\mathrm{i} \alpha} & 0
\end{array}\right)\left(\begin{array}{cc}
1 & -\mathrm{ie}^{\mathrm{i} \alpha} \psi^{-1}(k) \mathrm{e}^{2 \mathrm{i} t g_{+}(k)} \\
0 & 1
\end{array}\right) F_{-}^{\sigma_{3}}(k) .
\end{aligned}
$$

where $\left.\psi(k):=F_{+} k\right) F_{-}^{-1}(k)$. Similarly, if

$$
F_{-}(k) F_{+}(k)=-\mathrm{ie}^{\mathrm{i} \alpha} \bar{f}^{-1}(\bar{k}) \delta^{-2}(k) \text { for } k \in \bar{\gamma}_{g},
$$

then for $k \in \bar{\gamma}_{g}$ we can factorize $J^{(2)}(x, t ; k)$ as follows:

$$
\begin{aligned}
& J^{(2)}(x, t ; k):=\left(\begin{array}{cc}
\mathrm{e}^{-2 \mathrm{i} t g_{+}(k)} & -\bar{f}(\bar{k}) \delta^{2}(k) \\
0 & \mathrm{e}^{2 \mathrm{i} t g_{+}(k)}
\end{array}\right) \\
& =\left(\begin{array}{cc}
F_{+}^{-1}(k) & 0 \\
0 & F_{+}(k)
\end{array}\right)\left(\begin{array}{cc}
F_{+}(k) F_{-}^{-1}(k) \mathrm{e}^{-2 \mathrm{i} t g_{+}(k)} & \mathrm{ie} \mathrm{i}^{\mathrm{\alpha} \alpha} \\
0 & F_{-}(k) F_{+}^{-1}(k) \mathrm{e}^{2 \mathrm{i} t g_{+}(k)}
\end{array}\right)\left(\begin{array}{cc}
F_{-}(k) & 0 \\
0 & F_{-}^{-1}(k)
\end{array}\right) \\
& =F_{+}^{-\sigma_{3}}(k)\left(\begin{array}{cc}
1 & 0 \\
-\mathrm{i} \mathrm{e}^{-\mathrm{i} \alpha} \psi^{-1}(k) \mathrm{e}^{2 \mathrm{i} t g_{+}(k)} & 1
\end{array}\right)\left(\begin{array}{cc}
0 & \mathrm{ie}^{\mathrm{i} \alpha} \\
\mathrm{i} \mathrm{e}^{-\mathrm{i} \alpha} & 0
\end{array}\right)\left(\begin{array}{cc}
1 & 0 \\
-\mathrm{i} \mathrm{e}^{-\mathrm{i} \alpha} \psi(k) \mathrm{e}^{-2 \mathrm{i} t g_{+}(k)} & 1
\end{array}\right) F_{-}^{\sigma_{3}}(k) .
\end{aligned}
$$

This leads us to introduce the following scalar Riemann-Hilbert problem:

Scalar RH-problem. Find a scalar function $F(k)$ such that

- $F$ is analytic outside the contour $\gamma_{g} \cup \bar{\gamma}_{g}$,

- $F$ does not vanish,

- $F$ is bounded at infinity, 
- F satisfies the jump relation

$$
F_{-}(k) F_{+}(k)=h(k) \delta^{-2}(k), \quad k \in \gamma_{g} \cup \bar{\gamma}_{g},
$$

where

$$
h(k)= \begin{cases}-\mathrm{i} \mathrm{e}^{\mathrm{i} \alpha} f(k), & k \in \gamma_{g}, \\ -\mathrm{i} \mathrm{e}^{\mathrm{i} \alpha} \bar{f}^{-1}(\bar{k}), & k \in \bar{\gamma}_{g} .\end{cases}
$$

To solve this scalar RH problem let us use the function $X(k)=\sqrt{(k-E)(k-\bar{E})}$ welldefined on $\mathbb{C} \backslash \gamma_{g} \cup \bar{\gamma}_{g}$. Let $X_{+}(k)$ as usual be the limit of $X(\zeta), \zeta \notin \gamma_{g} \cup \bar{\gamma}_{g}$, as $\zeta \rightarrow k$ from the left side of $\gamma_{g} \cup \bar{\gamma}_{g}$. Since

$$
\left[\frac{\log F(k)}{X(k)}\right]_{+}-\left[\frac{\log F(k)}{X(k)}\right]_{-}=\frac{\log \left[h(k) \delta^{-2}(k)\right]}{X_{+}(k)}, \quad k \in \gamma_{g} \cup \bar{\gamma}_{g}
$$

the solution of the scalar $\mathrm{RH}$ problem is given by

$$
\begin{aligned}
& F(k)=\exp \left\{\frac{X(k)}{2 \pi \mathrm{i}} \int_{\gamma_{g} \cup \bar{\gamma}_{g}} \frac{\log \left[h(s) \delta^{-2}(s, \xi)\right]}{s-k} \frac{\mathrm{d} s}{X_{+}(s)}\right\} \\
& F(\infty)=\mathrm{e}^{\mathrm{i} \phi(\xi)} \text { with } \phi(\xi)=\frac{1}{2 \pi} \int_{\gamma_{g} \cup \bar{\gamma}_{g}} \log \left[h(s) \delta^{-2}(s, \xi)\right] \frac{\mathrm{d} s}{X_{+}(s)} .
\end{aligned}
$$

Putting this solution $F(k)$ in the above factorization of $J^{(2)}(x, t ; k)$ for $k \in \gamma_{g}$, and using

$$
\begin{gathered}
\left(\begin{array}{cc}
1 & \overline{\hat{\rho}}_{1+}(\bar{k}) \delta^{2}(k) \mathrm{e}^{-2 \mathrm{itg} g_{+}(k)} \\
0 & 1
\end{array}\right) F_{+}^{-\sigma_{3}}(k)=F_{+}^{-\sigma_{3}}(k)\left(\begin{array}{cc}
1 & \overline{\hat{\rho}}_{1+}(\bar{k}) \delta^{2}(k) F_{+}^{2}(k) \mathrm{e}^{-2 \mathrm{i} t g_{+}(k)} \\
0 & 1
\end{array}\right), \\
F_{-}^{\sigma_{3}}(k)\left(\begin{array}{cc}
1 & -\overline{\hat{\rho}}_{1-}(\bar{k}) \delta^{2}(k) \mathrm{e}^{2 \mathrm{i} t g_{+}(k)} \\
0 & 1
\end{array}\right)=\left(\begin{array}{cc}
1 & -\overline{\hat{\rho}}_{1-}(\bar{k}) \delta^{2}(k) F_{-}^{2}(k) \mathrm{e}^{2 i t g_{+}(k)} \\
0 & 1
\end{array}\right) F_{-}^{\sigma_{3}}(k),
\end{gathered}
$$

we get for $k \in \gamma_{g}$ :

$$
J^{(3)}(x, t ; k)=F_{+}^{-\sigma_{3}}(k) N_{\text {up }}(k) J^{\text {mod }} \hat{N}_{\text {up }}(k) F_{-}^{\sigma_{3}}(k),
$$

where

$$
\begin{aligned}
& N_{\text {up }}(k)=\left(\begin{array}{cc}
1 & \delta^{2}(k) F_{+}^{2}(k)\left[\hat{\hat{\rho}}_{1+}(\bar{k})+f^{-1}(k)\right] \mathrm{e}^{-2 \mathrm{i} t g_{+}(k)} \\
0 & 1
\end{array}\right), \\
& J^{\bmod }=\left(\begin{array}{cc}
0 & \mathrm{ie}^{\mathrm{i} \alpha} \\
\mathrm{ie}^{-\mathrm{i} \alpha} & 0
\end{array}\right) \text {, } \\
& \hat{N}_{\text {up }}(k)=\left(\begin{array}{cc}
1 & -\delta^{2}(k) F_{-}^{2}(k)\left[\hat{\hat{\rho}}_{1-}(\bar{k})-f^{-1}(k)\right] \mathrm{e}^{-2 \mathrm{i} t g_{-}(k)} \\
0 & 1
\end{array}\right) .
\end{aligned}
$$

Similarly, for $k \in \bar{\gamma}_{g}$, we get:

$$
J^{(3)}(x, t ; k)=F_{+}^{-\sigma_{3}}(k) N_{\text {low }}(k) J^{\bmod } \hat{N}_{\text {low }}(k) F_{-}^{\sigma_{3}}(k),
$$


where

$$
\begin{aligned}
& N_{\text {low }}(k)=\left(\begin{array}{cc}
1 & 0 \\
-\delta^{-2}(k) F_{+}^{-2}(k)\left[\hat{\rho}_{1+}(k)+\bar{f}^{-1}(\bar{k})\right] \mathrm{e}^{2 \mathrm{it} g_{+}(k)} & 1
\end{array}\right), \\
& J^{\mathrm{mod}}=\left(\begin{array}{cc}
0 & \mathrm{ie} \mathrm{i}^{\mathrm{i} \alpha} \\
\mathrm{i} \mathrm{e}^{-\mathrm{i} \alpha} & 0
\end{array}\right), \\
& \hat{N}_{\text {low }}(k)=\left(\begin{array}{cc}
1 & 0 \\
\delta^{-2}(k) F_{-}^{-2}(k)\left[\hat{\rho}_{1-}(k)-\bar{f}^{-1}(\bar{k})\right] \mathrm{e}^{2 \mathrm{i} t g_{-}(k)} & 1
\end{array}\right) .
\end{aligned}
$$

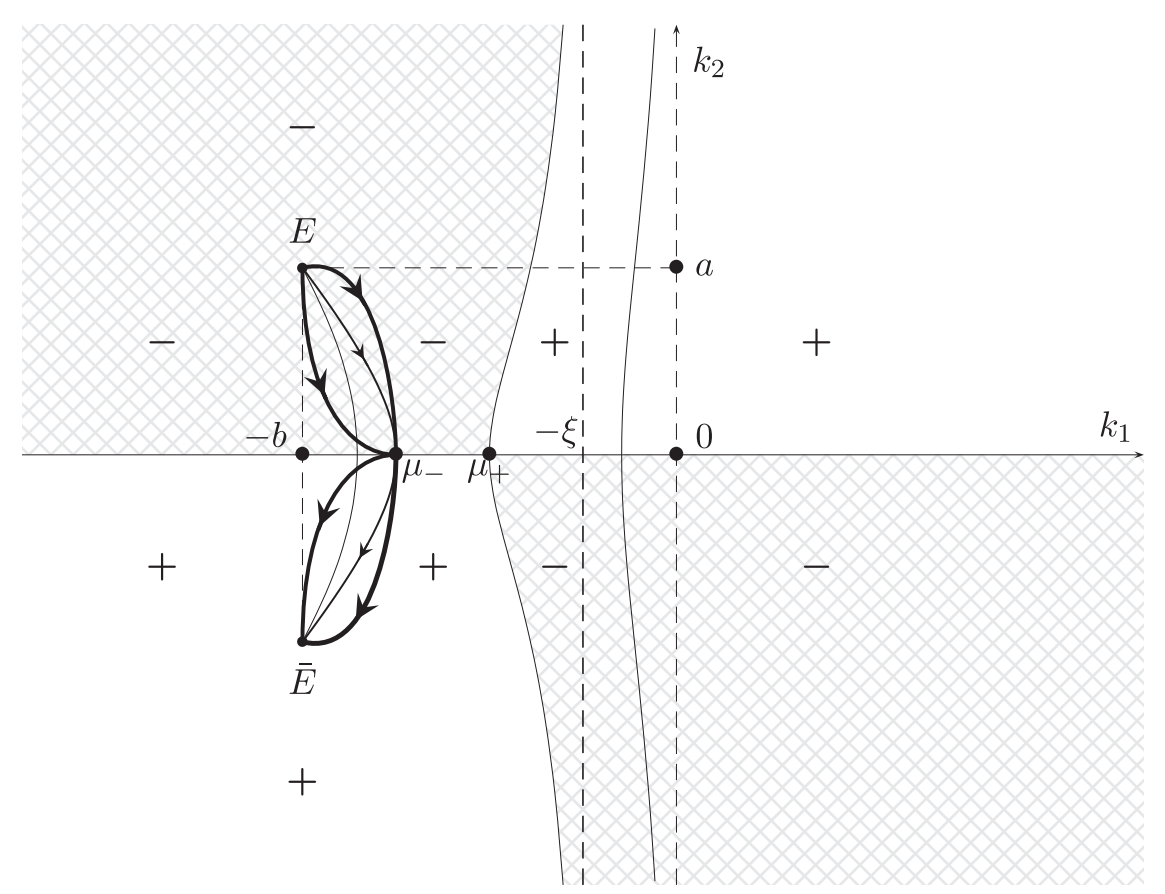

FiguRE 9. The lenses around $\gamma_{g} \cup \bar{\gamma}_{g}$ for $0<\xi<b-a \sqrt{2}$

5.4.5. The next step - the "opening lenses" step - is as follows (cf. [10]). Let

$$
M^{(4)}(x, t ; k):= \begin{cases}F^{\sigma_{3}}(\infty) M^{(3)}(x, t ; k) F^{-\sigma_{3}}(k) & k \text { outside the lenses, } \\ F^{\sigma_{3}}(\infty) M^{(3)}(x, t ; k) F^{-\sigma_{3}}(k) N_{\text {up }}(k) & k \text { inside the upper right lens, } \\ F^{\sigma_{3}}(\infty) M^{(3)}(x, t ; k) F^{-\sigma_{3}}(k) \hat{N}_{\text {up }}^{-1}(k) & k \text { inside the upper left lens, } \\ F^{\sigma_{3}}(\infty) M^{(3)}(x, t ; k) F^{-\sigma_{3}}(k) N_{\text {low }}(k) & k \text { inside the lower right lens, } \\ F^{\sigma_{3}}(\infty) M^{(3)}(x, t ; k) F^{-\sigma_{3}}(k) \hat{N}_{\text {low }}^{-1}(k) & k \text { inside the lower left lens. }\end{cases}
$$

Here we again use property \#1 to perform the analytic continuation of the matrices $N_{\text {up }}(k)$, $N_{\text {low }}(k), \hat{N}_{\text {up }}(k)$ and $\hat{N}_{\text {low }}(k)$ to the indicated domains. Then we have

$$
M_{-}^{(4)}(x, t ; k)=M_{+}^{(4)}(x, t ; k) J^{(4)}(x, t ; k),
$$


where

$$
J^{(4)}(x, t ; k)= \begin{cases}N_{\text {up }}(x, t ; k) & \text { on the boundary of the right upper lens, } \\ \hat{N}_{\text {up }}(x, t ; k) & \text { on the boundary of the left upper lens, } \\ J^{\text {mod }} & k \in \gamma_{g} \cup \bar{\gamma}_{g} \\ N_{\text {low }}(x, t ; k) & \text { on the boundary of the right lower lens, } \\ \hat{N}_{\text {low }}(x, t ; k) & \text { on the boundary of the left lower lens. }\end{cases}
$$

Due to the signature table of the phase function $g(k)$ the matrices $N_{\text {up }}(x, t ; k), \hat{N}_{\text {up }}(x, t ; k)$, $N_{\text {low }}(x, t ; k), \hat{N}_{\text {low }}(x, t ; k)$ are exponentially close to the unit matrix outside of small neighborhoods of the end points $E$ and $\bar{E}$ and of the stationary phase point $\mu_{-}$. The analysis of parametrix solutions near the points $E, \bar{E}$ and the point $\mu_{-}$is very similar to the analysis done in [9] and [8], respectively. In the first case, the relevant model Riemann-Hilbert problem is solvable in terms of Bessel functions while in the second case this is again a parabolic cylinder Riemann-Hilbert problem. Skipping technical details, we arrive at the following asymptotic representation of the function $M^{(4)}(x, t ; k)$,

$$
M^{(4)}(x, t ; k)=\left(I+\mathrm{O}\left(t^{-1 / 2}\right)\right) M^{\bmod }(x, t ; k),
$$

where $M^{\bmod }(x, t ; k)$ solves the 0 -gap model problem $\mathrm{RH}^{\text {mod }}$ (cf. [9]):

\section{Zero-gap model RH-problem.}

$$
M_{-}^{\bmod }(x, t ; k)=M_{+}^{\bmod }(x, t ; k) J^{\bmod }, \quad k \in \gamma_{g} \cup \bar{\gamma}_{g},
$$

with constant jump matrix:

$$
J^{\bmod }=\left(\begin{array}{cc}
0 & \mathrm{ie}^{\mathrm{i} \alpha} \\
\mathrm{ie}^{-\mathrm{i} \alpha} & 0
\end{array}\right) .
$$

Let us remind the multi-valued function

$$
\nu(k)=\left(\frac{k-E}{k-\bar{E}}\right)^{1 / 4}=\left(\frac{k+b-\mathrm{i} a}{k+b+\mathrm{i} a}\right)^{1 / 4}
$$

introduced in (1.8f), $\S 1$, and such that

$$
\nu(k)=1-\frac{\mathrm{i} a}{2 k}+\mathrm{O}\left(\frac{1}{k^{2}}\right) \text { as } k \rightarrow \infty .
$$

Since $\nu_{-}(k)=\mathrm{i} \nu_{+}(k)$ on the cut $\gamma_{g} \cup \bar{\gamma}_{g}$ the solution of $\mathrm{RH}^{\text {mod }}$ is explicitly given by

$$
M^{\bmod }(x, t ; k)=\frac{1}{2}\left(\begin{array}{cc}
\nu(k)+\frac{1}{\nu(k)} & \mathrm{e}^{\mathrm{i} \alpha}\left(\nu(k)-\frac{1}{\nu(k)}\right) \\
\mathrm{e}^{-\mathrm{i} \alpha}\left(\nu(k)-\frac{1}{\nu(k)}\right) & \nu(k)+\frac{1}{\nu(k)}
\end{array}\right) .
$$

Let $M^{\bullet}(x, t ; k)$ denotes the solution of the Riemann-Hilbert-problem $\mathrm{RH}^{\bullet}$ and

$$
m_{12}^{\bullet}(x, t):=\lim _{k \rightarrow \infty}\left(k M^{\bullet}(x, t ; k)\right)_{12} .
$$


The previous considerations yield the following chain of equalities:

$$
\begin{aligned}
q(x, t) & =2 \mathrm{i} m_{12}(x, t) \\
& =2 \mathrm{ie}^{2 \mathrm{i} t g_{\infty}(\xi)} m_{12}^{(1)}(x, t) \\
& =2 \mathrm{ie}^{2 \mathrm{i} t g_{\infty}(\xi)} m_{12}^{(2)}(x, t) \\
& =2 \mathrm{ie}^{2 \mathrm{i} t g_{\infty}(\xi)} m_{12}^{(3)}(x, t)+\mathrm{O}\left(t^{-1 / 2}\right) \\
& =2 \mathrm{ie}^{2 \mathrm{i} t g_{\infty}(\xi)} m_{12}^{(4)}(x, t) F^{-2}(\infty)+\mathrm{O}\left(t^{-1 / 2}\right) \\
& =2 \mathrm{ie}^{2 \mathrm{i} t g_{\infty}(\xi)} m_{12}^{\bmod }(x, t) F^{-2}(\infty)+\mathrm{O}\left(t^{-1 / 2}\right) .
\end{aligned}
$$

Taking into account that $g_{\infty}(\xi)=\omega+4 b \xi, 2 \mathrm{i} m_{12}^{\bmod }(x, t) \equiv a \mathrm{e}^{\mathrm{i} \alpha}, F^{-2}(\infty)=\mathrm{e}^{-2 \mathrm{i} \phi(\xi)}$ we get the following theorem:

Theorem 4 (plane wave region, $0 \leq \xi<b-a \sqrt{2}$ ). Suppose that all conditions of Theorem 1 and conditions \#1, \#2, \#3 are satisfied.

Then in the region $0 \leq \xi<b-a \sqrt{2}$ the solution (4.2) of the IBV problem takes the form of a plane wave:

$$
\begin{aligned}
& q(x, t)=a \mathrm{e}^{\mathrm{i} \alpha} \mathrm{e}^{2 \mathrm{i}[b x+\omega t-\phi(\xi)]}+\mathrm{O}\left(t^{-1 / 2}\right), \quad t \rightarrow+\infty, \\
& \phi(\xi)=\frac{1}{2 \pi} \int_{\gamma_{g} \cup \bar{\gamma}_{g}} \log \left[h(s) \delta^{-2}(s, \xi)\right] \frac{\mathrm{d} s}{X_{+}(s)} .
\end{aligned}
$$

Remark 2. As it is indicated, the above arguments are valid in the case $x=0$, i.e. $\xi=0$ as well. This, in particular, implies the relation,

$$
\phi(0)=0,
$$

which is the constraint (2.23).

Remark 3. If $b \leq a \sqrt{2}$ then the plane wave region is empty.

Remark 4. Equation (5.11) is consistent with our assumption (1.5) on the structure of the Dirichlet to Neumann map. It also should be noticed that in the case $x=0$, i.e., $\xi=0$, we do not need restrictions $\# 1$ and \#2 on the $\mathrm{RH}$ data to proceed with our asymptotic approach. Indeed, in this case, $\gamma=\gamma_{g}$ and therefore no deformation of the original contour $\gamma \cup \bar{\gamma}$ is needed.

5.5. Modulated elliptic region $\boldsymbol{b}-\boldsymbol{a} \sqrt{\mathbf{2}}<\boldsymbol{\xi} \equiv \frac{\boldsymbol{x}}{\mathbf{4} \boldsymbol{t}}<\boldsymbol{b}$. When $\xi=\xi_{0}=b-a \sqrt{2}$ the zeros $\mu_{-}\left(\xi_{0}\right)$ and $\mu_{+}\left(\xi_{0}\right)$, see (5.9), of the differential $\mathrm{d} g(k)$ coincide (See Fig. 10):

$$
\mu_{-}\left(\xi_{0}\right)=\mu_{+}\left(\xi_{0}\right)=\mu=-\frac{b+\xi_{0}}{2} .
$$

For $\xi>\xi_{0}$ the zeros $\mu_{ \pm}(\xi)$ of $\mathrm{d} g(k)$ become complex conjugate. As a result the previous considerations fail. We need to introduce a new $g$-function for this region.

Let $\xi>\xi_{0}$. We start by replacing the original $\mathrm{RH}$ problem by the analogous $\mathrm{RH}$ problem whose phase function is the previous $g$-function $g_{0}=g\left(\xi_{0}\right)$ associated to $\xi=\xi_{0}$. The contour is

$$
\Sigma=\mathbb{R} \cup \Gamma \cup \bar{\Gamma} \cup \gamma_{0} \cup \bar{\gamma}_{0},
$$




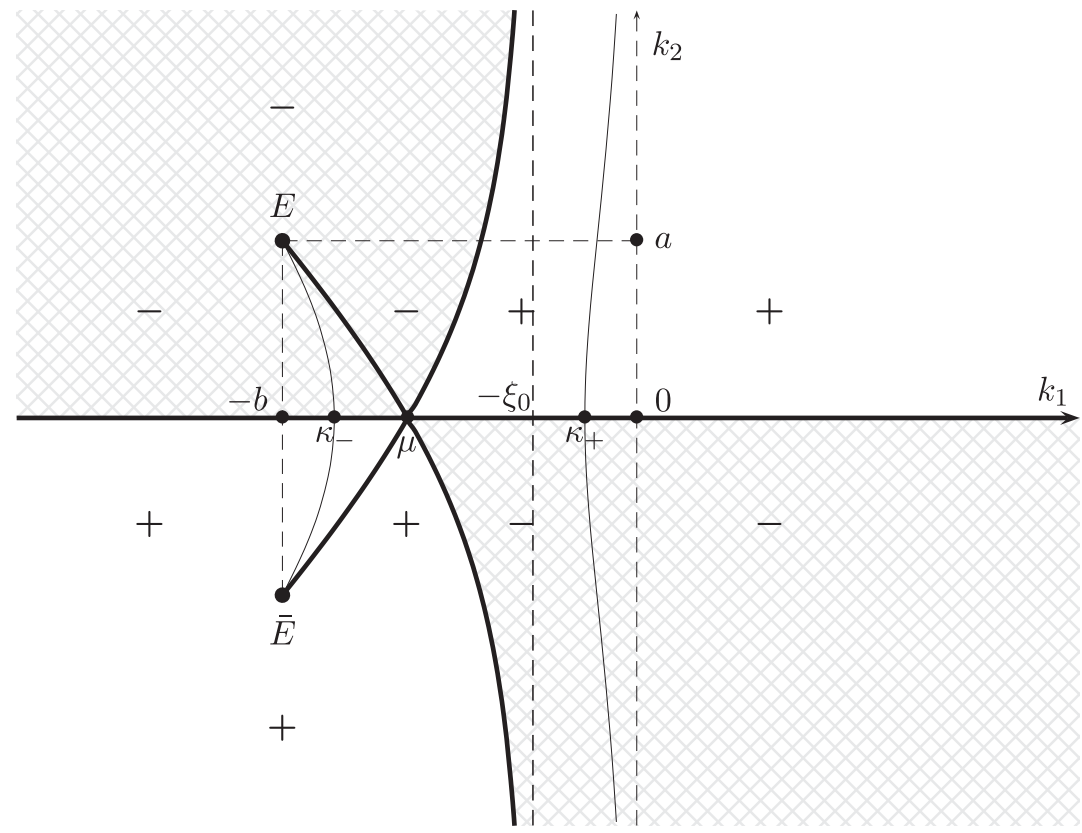

Figure 10. The signature table of $\operatorname{Im} g(k)$ for $\xi=\xi_{0}=b-a \sqrt{2}$

where $\gamma_{0}:=\gamma_{g\left(\xi_{0}\right)}$ and $\bar{\gamma}_{0}:=\bar{\gamma}_{g\left(\xi_{0}\right)}$. As before this new RH problem is equivalent to the original one.

5.5.1. The new $g$-function. A suitable $g$-function for $\xi>\xi_{0}$ can be obtained as follows. First, we need to introduce a new real stationary point $\mu=\mu(\xi)$ which must be a zero of the new differential $\mathrm{d} g$. On the other hand we have to preserve the asymptotic behavior of the $g$-function for large $k$. To do so we must change the denominator of the differential $\mathrm{d} g$. Thus the new differential takes the form:

$$
\mathrm{d} g(k)=4 \frac{(k-\mu(\xi))\left(k-\mu_{-}(\xi)\right)\left(k-\mu_{+}(\xi)\right)}{\sqrt{(k-E)(k-\bar{E})(k-d(\xi))(k-\bar{d}(\xi))}} \mathrm{d} k
$$

where $\mu(\xi), \mu_{ \pm}(\xi)$, and $d(\xi)$ are to be determined. It is easy to see that if we choose

$$
\mu\left(\xi_{0}\right)=\mu_{ \pm}\left(\xi_{0}\right)=d\left(\xi_{0}\right)=\bar{d}\left(\xi_{0}\right)=-\frac{b+\xi_{0}}{2},
$$

then for $\xi=\xi_{0}$ the new differential coincides with the previous one:

$$
\mathrm{d} g(k)=4 \frac{\left(k-\mu_{-}\right)\left(k-\mu_{+}\right)}{X(k)} \mathrm{d} k .
$$


One can also see that $\mathrm{d} g$ is an Abelian (elliptic) differential of the second kind with poles at $\infty_{ \pm}$on the Riemann surface $\mathcal{X}$ of

$$
\begin{aligned}
& w(k)=\sqrt{(k-E)(k-\bar{E})(k-d(\xi))(k-\bar{d}(\xi))}, \\
& E=-b+\mathrm{i} a \\
& d(\xi)=d_{1}(\xi)+\mathrm{i} d_{2}(\xi) .
\end{aligned}
$$

The branch of the square root is fixed by his asymptotics on the upper sheet $\mathcal{X}_{+}$:

$$
w(k)=k^{2}+\mathrm{O}(k), \quad k \rightarrow \infty_{+} .
$$

We choose on this Riemann surface a basis $\{\mathfrak{a}, \mathfrak{b}\}$ of cycles as follows. The $\mathfrak{b}$-cycle is a closed clock-wise oriented simple loop around the arc $\gamma_{E, d}$ joining $E$ and $d$. The a-cycle starts on the upper sheet $\mathcal{X}_{+}$from the left side of the cut $\gamma_{E, d}$, goes to the left side of the cut $\gamma_{\bar{d}, \bar{E}}$, proceeds to the lower sheet $\mathcal{X}_{-}$, and then returns to the starting point.

We write the Abelian differential $\mathrm{d} g(k)$ in the form:

$$
\mathrm{d} g(k)=4 \frac{k^{3}+c_{2} k^{2}+c_{1} k+c_{0}}{w(k)} \mathrm{d} k
$$

and normalize it so that its $\mathfrak{a}$-period vanishes. Since

$$
\int_{\mathfrak{a}} \mathrm{d} g=2 \int_{\bar{d}}^{d} \mathrm{~d} g
$$

where the path of integration is the line segment $[\bar{d}, d]$, this normalization condition means

$$
c_{0}=-\frac{\int_{\bar{d}}^{d}\left(k^{3}+c_{2} k^{2}+c_{1} k\right) \frac{\mathrm{d} k}{w(k)}}{\int_{\bar{d}}^{d} \frac{\mathrm{d} k}{w(k)}} .
$$

Note that $c_{0}$ is real. It depends on $c_{1}, c_{2}, d_{1}(\xi)$ and $d_{2}(\xi)$. The requirement

$$
g(k)=\theta(k)+\mathrm{O}(1) \text { as } k \rightarrow \infty_{+},
$$

i.e. $g(k)=2 k^{2}+4 \xi k+\mathrm{O}(1)$, implies

$$
\begin{aligned}
& c_{2}=b+\xi-d_{1}, \\
& c_{1}=b \xi-(b+\xi) d_{1}+\frac{1}{2}\left(d_{2}^{2}+a^{2}\right) .
\end{aligned}
$$

Thus, $c_{0}=c_{0}\left(\xi, d_{1}, d_{2}\right)$. Then the $g$-function, taken as the sum of two Abelian integrals:

$$
g(k)=2\left(\int_{E}^{k}+\int_{\bar{E}}^{k}\right) \frac{z^{3}+c_{2} z^{2}+c_{1} z+c_{0}}{w(z)} \mathrm{d} z,
$$


has real $\mathfrak{b}$-period ${ }^{1}$

$$
B_{g}=2\left(\int_{E}^{d}+\int_{\bar{E}}^{\bar{d}}\right) \frac{z^{3}+c_{2} z^{2}+c_{1} z+c_{0}}{w(z)} \mathrm{d} z
$$

and the following asymptotics at $k=\infty_{+}$:

$$
g(k)=2 k^{2}+4 \xi k+g_{\infty}(\xi)+\mathrm{O}\left(k^{-1}\right),
$$

where

$$
g_{\infty}(\xi)=2\left(\int_{E}^{\infty_{+}}+\int_{\bar{E}}^{\infty+}\right)\left[\frac{z^{3}+c_{2} z^{2}+c_{1} z+c_{0}}{w(z)}-(z+\xi)\right] \mathrm{d} z+2 a^{2}-2 b^{2}+4 b \xi
$$

is a real-valued function of $\xi$.

Convention (integration paths). The contours of integration in both integrals in (5.12), (5.13) and (5.14) are chosen according to the following convention that allows to work with a single-valued branch of the multi-valued function $g(k)$. In what follows we use only the upper sheet of the Riemann surface. Moreover, let us complete the contour $\gamma_{E, d} \cup \overline{\gamma_{E, d}} \cup[d, \bar{d}]$ by attaching to it the infinite vertical pieces, $(-b+\mathrm{i} \infty, E]$ and $[\bar{E},-b-\mathrm{i} \infty)$. Then the integration paths are chosen so that they do not intersect the augmented contour. Observe also, that across the added pieces, the function $g(k)$ does not jump:

$$
g_{+}(k)-g_{-}(k)=0 \text { for } k \in(-b+\mathrm{i} \infty, E) \cup(\bar{E},-b-\mathrm{i} \infty) .
$$

In order to determine $\mu, \mu_{ \pm}, d$ and $\bar{d}$ as functions of $\xi$ let us rewrite the differential $\mathrm{d} g$ in the form:

$$
\mathrm{d} g(k)=4 \frac{(k-\mu)\left(k-\mu_{-}\right)\left(k-\mu_{+}\right)}{w(k)} \mathrm{d} k,
$$

where $\mu_{ \pm}=\mu_{1} \pm \mathrm{i} \mu_{2}$ and $\mu, \mu_{1}$ are negative. Comparing with the previous form of the differential $\mathrm{d} g$ we obtain

$$
\begin{aligned}
& \mu+2 \mu_{1}-d_{1}=-(b+\xi) \\
& 2 \mu \mu_{1}+\mu_{1}^{2}+\mu_{2}^{2}+(b+\xi) d_{1}-\frac{1}{2} d_{2}^{2}=\frac{1}{2} a^{2}+b \xi \\
& \mu\left(\mu_{1}^{2}+\mu_{2}^{2}\right)=-c_{0}\left(\xi, d_{1}, d_{2}\right) .
\end{aligned}
$$

Let $(k-d)^{1 / 2}$ be the local parameter at $d$. It is easy to see that the local expansion of $g(k)$ at $d$ is of the form

$$
g(k)=B_{g}+g_{1}(k-d)^{1 / 2}+g_{2}(k-d)^{3 / 2}+\ldots, \quad B_{g} \in \mathbb{R} .
$$

\footnotetext{
${ }^{1}$ Taking into account the relation $\int_{\bar{d}}^{d} \mathrm{~d} g=0$ and the absence of residue of $\mathrm{d} g$ at $k=\infty_{ \pm}$we see that in fact $\int_{\bar{E}}^{E} \mathrm{~d} g=0$ as well, and that the function $g(k)$ can be written as a single Abelian integral:

$$
g(k)=4 \int_{E}^{k} \frac{z^{3}+c_{2} z^{2}+c_{1} z+c_{0}}{w(z)} \mathrm{d} z,
$$

and, simultaneously, we have indeed

$$
B_{g}=\int_{\mathfrak{b}} \mathrm{d} g
$$
}


i. Since $g(E)=\operatorname{Im} g(d)=0$ then the points $E$ and $d=d(\xi)$ are connected by a curve $\gamma_{d}$ where $\operatorname{Im} g(k) \equiv 0$.

ii. Since $g(k)$ is real on the real axis then there exist a real point $\mu=\mu(\xi)$ and some curve connecting $\mu$ and $d$, where $\operatorname{Im} g(k) \equiv 0$.

iii. Since $g(k)$ behaves like $\theta(k)$ for large $k$ then there exists a curve where $\operatorname{Im} g(k) \equiv 0$, starting from $d$ and going to infinity along the asymptotic line $\operatorname{Re} k=-\xi$. Here $-\xi$ is the stationary point of the phase function $\theta(k)$.

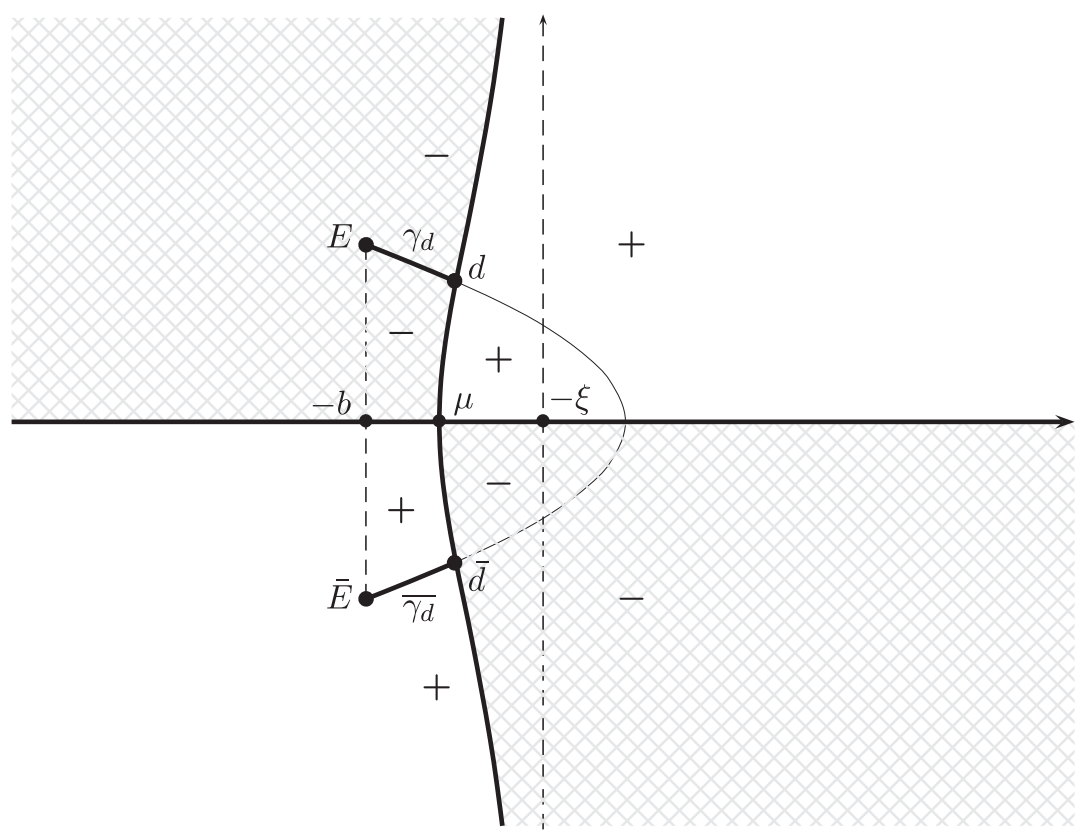

Figure 11. The signature table of $\operatorname{Im} g(k)$ in the region $b-a \sqrt{2}<\xi<b$

Thus the curve $\operatorname{Im} g(k)=0$ must have three branches going out from the point $d$. This is possible if and only if $g_{1}=0$, i.e.

$$
\left.(k-d)^{1 / 2} g^{\prime}(k)\right|_{k=d}=4 \frac{(d-\mu(\xi))\left(d-\mu_{-}(\xi)\right)\left(d-\mu_{+}(\xi)\right)}{\sqrt{(k-E)(k-\bar{E})(d-\bar{d})}}=0 .
$$

Since $\mu$ is real then $\mu_{+}=d$ and $\mu_{-}=\bar{d}$ and finally we have

$$
\mathrm{d} g(k)=4(k-\mu(\xi)) \sqrt{\frac{(k-d(\xi))(k-\bar{d}(\xi))}{(k-E)(k-\bar{E})}} \mathrm{d} k .
$$

Previous considerations yield the signature table of the function $\operatorname{Im} g(k)$ shown in Fig. 11. 
The functions $\mu(\xi), d(\xi)=d_{1}(\xi)+\mathrm{i} d_{2}(\xi)$ have to satisfy now the following equations:

$$
\begin{aligned}
& \mu+d_{1}=-(b+\xi), \\
& d_{2}^{2}-2\left(d_{1}+\frac{b+\xi}{2}\right)^{2}=a^{2}-\frac{(b-\xi)^{2}}{2}, \\
& \mu\left(\mu_{1}^{2}+\mu_{2}^{2}\right)=-c_{0}\left(\xi, d_{1}, d_{2}\right) .
\end{aligned}
$$

These three equations can be reduced to one equation with respect to $\mu(\xi)$ involving elliptic integrals. Indeed, let us put

$$
d_{1}=d_{1}(\xi)=-\mu-b-\xi,
$$

then

$$
d_{2}=d_{2}(\xi)=\sqrt{2 \mu^{2}+2(b+\xi) \mu+a^{2}+2 b \xi} .
$$

The $\mathfrak{a}$-period of the differential $\mathrm{d} g$ vanishes. It means that

$$
\mu=\frac{I_{1}\left(d_{1}, d_{2}\right)}{I_{0}\left(d_{1}, d_{2}\right)}
$$

where

$$
\begin{aligned}
& I_{1}\left(d_{1}, d_{2}\right)=\int_{d_{1}-\mathrm{i} d_{2}}^{d_{1}+\mathrm{i} d_{2}} z \sqrt{\frac{\left(z-d_{1}\right)^{2}+d_{2}^{2}}{(z+b)^{2}+a^{2}}} \mathrm{~d} z, \\
& I_{0}\left(d_{1}, d_{2}\right)=\int_{d_{1}-\mathrm{i} d_{2}}^{d_{1}+\mathrm{i} d_{2}} \sqrt{\frac{\left(z-d_{1}\right)^{2}+d_{2}^{2}}{(z+b)^{2}+a^{2}}} \mathrm{~d} z .
\end{aligned}
$$

Thus we have one functional equation for $\mu=\mu(\xi)$ :

$$
\begin{aligned}
& \mu=H(\mu, \xi), \\
& H(\mu, \xi)=\frac{I_{1}\left(-\mu-b-\xi, \sqrt{2 \mu^{2}+2(b+\xi) \mu+a^{2}+2 b \xi}\right)}{I_{0}\left(-\mu-b-\xi, \sqrt{2 \mu^{2}+2(b+\xi) \mu+a^{2}+2 b \xi}\right)} .
\end{aligned}
$$

We are interested in the solution $\mu=\mu(\xi)$ which moves into the closed interval $[-b,-b+$ $a / \sqrt{2}]$, when $\xi$ belongs to $[b, b-a \sqrt{2}]$. Equation (5.15) is consistent with this requirement.

Indeed, if $\xi=b$ then by construction of the $g$-function the branch point $d(b)=d_{1}(b)+\mathrm{i} d_{2}(b)$ must coincide with the branch point $E=-b+\mathrm{i} a$. In this case the elliptic integrals degenerate and $I_{0}=2 \mathrm{i} a, I_{1}=-2 \mathrm{i} a b$. Hence $\mu(b)=-b$. The function $g(k)$ reduces consequently (up to a constant) to the phase function $\theta(k)=\theta(k, \xi)=2 k^{2}+4 \xi k$ which attended the problem in the region $\xi>b$ :

$$
g(k, b)-2|E|^{2}=\theta(k, b) .
$$

On the other hand, let us note that after the change of variable $z=d_{1}+\mathrm{i} s d_{2}$ in the integrals $I_{0}$ and $I_{1}$ the functional equation takes the form:

$$
\mu=d_{1}-d_{2} \frac{\int_{-1}^{1} \frac{s \sqrt{1-s^{2}} \mathrm{~d} s}{\sqrt{a^{2}+\left(b+d_{1}+\mathrm{i} s d_{2}\right)^{2}}}}{\int_{-1}^{1} \frac{\sqrt{1-s^{2}} \mathrm{~d} s}{\sqrt{a^{2}+\left(b+d_{1}+\mathrm{i} s d_{2}\right)^{2}}}}
$$


with the substitution:

$$
\begin{aligned}
& d_{1}:=d_{1}(\mu, \xi)=-\mu-b-\xi \\
& d_{2}:=d_{2}(\mu, \xi)=\sqrt{2 \mu^{2}+2(b+\xi) \mu+a^{2}+2 b \xi} .
\end{aligned}
$$

If $\xi=\xi_{0}=b-a \sqrt{2}$ then, due to the construction, $d_{2}\left(\xi_{0}\right)$ must be equal to zero and, hence, $d_{1}\left(\xi_{0}\right)=-\left(b+\xi_{0}\right) / 2=-b+a / \sqrt{2}$. Therefore $\mu\left(\xi_{0}\right)=d_{1}\left(\xi_{0}\right)=-b+a / \sqrt{2}$. The function $g(k)$ reduces consequently to the $g$-function considered in the previous subsection $\S 5.4$ for the region $0<\xi<b-a \sqrt{2}$.

For $b-a \sqrt{2}<\xi<b$ we deform the contour $\gamma_{0} \cup \bar{\gamma}_{0}$ into the contour $\gamma_{d} \cup \overline{\gamma_{d}} \cup[d, \bar{d}]$ (again using property \#1 of $c(k))$. The part $\gamma_{d} \cup \overline{\gamma_{d}}$ of this contour is chosen in such a way that $\operatorname{Im} g(k) \equiv 0$ on it. Then the function $g(k)$ has the following properties:

$$
\begin{aligned}
& g_{+}(k)+g_{-}(k)=0, \quad k \in \gamma_{d} \cup \overline{\gamma_{d}} ; \\
& g_{+}(k)-g_{-}(k)=B_{g}, \quad \operatorname{Im} B_{g}=0, \quad k \in[d, \bar{d}] .
\end{aligned}
$$

We remind that the function $g(k)$ is continuous in $k \in(-b+\mathrm{i} \infty, E) \cup(\bar{E},-b-\mathrm{i} \infty)$ :

$$
g_{+}(k)-g_{-}(k)=0, \quad k \in(-b+\mathrm{i} \infty, E) \cup(\bar{E},-b-\mathrm{i} \infty) .
$$

5.5.2. Let us perform the same transformations as in $\S \S 5.4 .2-5.4 .4$ :

$$
M(x, t ; k) \rightsquigarrow M^{(1)}(x, t ; k) \rightsquigarrow M^{(2)}(x, t ; k) \rightsquigarrow M^{(3)}(x, t ; k) .
$$

The function $\delta(k)$ is defined as (5.2), but now $\kappa_{0}=\mu(\xi)$, where $\mu(\xi)$ is the real stationary point of the new phase function $g(k)$. The RH problem is considered now on the contour $\Sigma^{(3)}$, depicted in Fig. 12. The jump matrix

$$
J^{(3)}(x, t ; k):=G_{+}^{-1}(k) J^{(2)}(x, t ; k) G_{-}(k)
$$

admit the following estimates as $t \rightarrow+\infty$ :

$$
\begin{aligned}
& J^{(3)}(x, t ; k)=I+\mathrm{O}\left(\mathrm{e}^{-\varepsilon t}\right), \quad \varepsilon>0, \\
& k \in \Sigma^{(3)} \backslash\left\{C_{\mu} \cap \Sigma^{(3)}\right\}, \quad \arg (k-\mu(\xi))=\frac{2 j-1}{4} \pi, \quad j=1,2,3,4,
\end{aligned}
$$

where $C_{\mu}$ is a small circle centered at $\mu$. Furthermore, for $k \in[d, \bar{d}]$ the jump matrix

$$
J^{(3)}(x, t ; k):=G_{+}^{-1}(k) J^{(2)}(x, t ; k) G_{-}(k)
$$

takes different forms:

- For $k \in[d, \bar{d}]$ and $\arg (k-\mu)>\pi / 4$,

$$
\left(\begin{array}{cc}
\mathrm{e}^{-2 \mathrm{i} t B_{g}} & 0 \\
f(k) \delta^{-2}(k) \mathrm{e}^{\mathrm{i} t\left(g_{+}(k)+g_{-}(k)\right)} & \mathrm{e}^{2 \mathrm{i} t B_{g}}
\end{array}\right) .
$$

- For $k \in[d, \bar{d}]$ and $0<\arg (k-\mu)<\pi / 4$,




- For $k \in[d, \bar{d}]$ and $-\pi / 4<\arg (k-\mu)<0$,

$\left(\begin{array}{cc}1 & -\overline{\hat{\rho}}_{+}(\bar{k}) \delta^{2}(k) \mathrm{e}^{-2 \mathrm{i} t g_{+}(k)} \\ 0 & 1\end{array}\right)\left(\begin{array}{cc}\mathrm{e}^{-2 \mathrm{i} t B_{g}} & -\bar{f}(\bar{k}) \delta^{2}(k) \mathrm{e}^{-\mathrm{i} t\left(g_{+}(k)+g_{-}(k)\right)} \\ 0 & \mathrm{e}^{2 \mathrm{i} t B_{g}}\end{array}\right)\left(\begin{array}{cc}1 & \overline{\hat{\rho}}_{-}(\bar{k}) \delta^{2}(k) \mathrm{e}^{-2 \mathrm{i} t g_{-}(k)} \\ 0 & 1\end{array}\right)$.

- For $k \in[d, \bar{d}]$ and $\arg (k-\mu)<-\pi / 4$,

$$
\left(\begin{array}{cc}
\mathrm{e}^{-2 \mathrm{i} t B_{g}} & -\bar{f}(\bar{k}) \delta^{2}(k) \mathrm{e}^{-\mathrm{i} t\left(g_{+}(k)+g_{-}(k)\right)} \\
0 & \mathrm{e}^{2 \mathrm{i} t B_{g}}
\end{array}\right) .
$$

Therefore, away from $d$ and $\bar{d}$, and for $t \rightarrow+\infty$, they are close to the diagonal matrix

$$
J^{\bmod }=\left(\begin{array}{cc}
\mathrm{e}^{-2 \mathrm{i} t B_{g}} & 0 \\
0 & \mathrm{e}^{2 \mathrm{i} t B_{g}}
\end{array}\right) .
$$

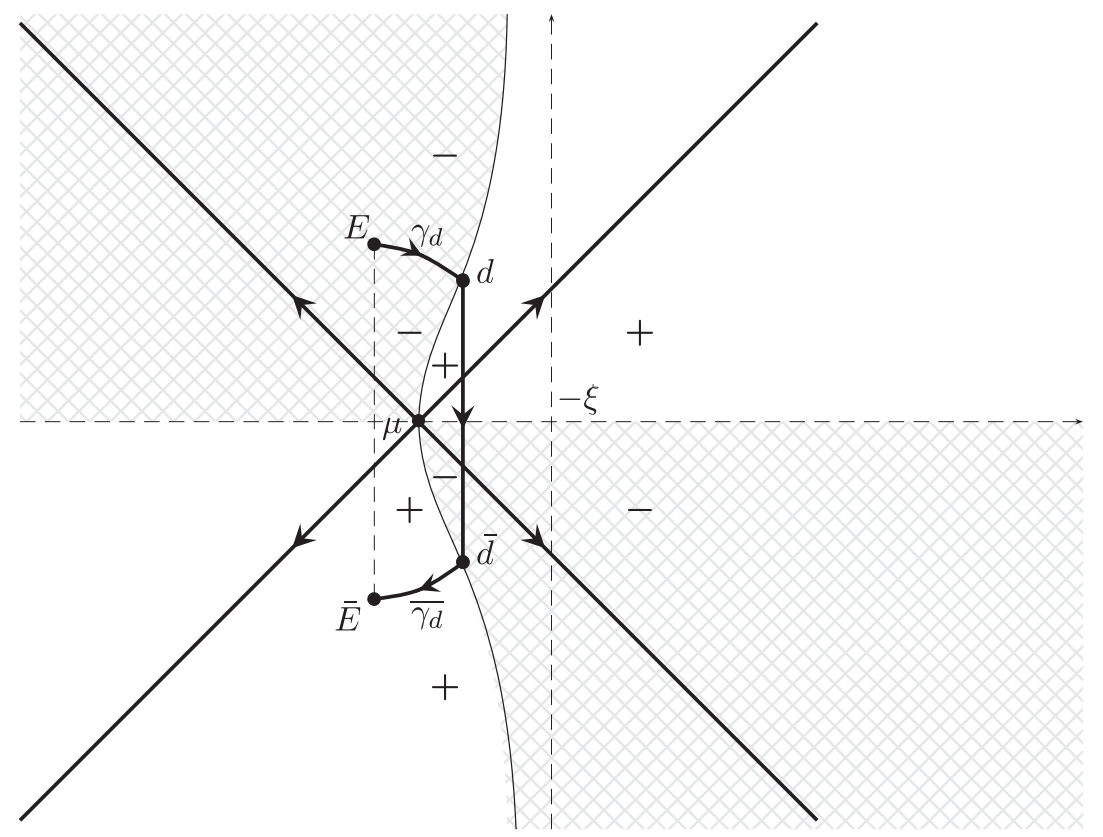

FiguRE 12. The contour $\Sigma^{(3)}$ for the elliptic region $b-a \sqrt{2}<\xi<b$

The jump matrix which has to be factorized is the part of the matrix $J^{(3)}(x, t ; k)$ on the arcs $\gamma_{d}$ and $\overline{\gamma_{d}}$ :

$$
J^{(3)}(x, t ; k)=\left\{\begin{array}{cc}
\left(\begin{array}{cc}
\mathrm{e}^{-2 \mathrm{i} t g_{+}(k)} & 0 \\
f(k) \delta^{-2}(k) & \mathrm{e}^{2 \mathrm{it} g_{+}(k)}
\end{array}\right), & k \in \gamma_{d}, \\
\left(\begin{array}{cc}
\mathrm{e}^{-2 \mathrm{i} t g_{+}(k)} & -\bar{f}(\bar{k}) \delta^{2}(k) \\
0 & \mathrm{e}^{2 \mathrm{i} t g_{+}(k)}
\end{array}\right), \quad k \in \overline{\gamma_{d}} .
\end{array}\right.
$$

As in $§ 5.4 .4$ we have to consider an auxiliary scalar Riemann-Hilbert problem:

Scalar RH-problem. Find a scalar function $F(k)$ such that 
- $F$ is analytic outside the contour $\gamma_{d} \cup \overline{\gamma_{d}}$,

- $F$ does not vanish,

- $F$ satisfies the jump relation

$$
F_{-}(k) F_{+}(k)=h(k) \delta^{-2}(k), \quad k \in \gamma_{d} \cup \overline{\gamma_{d}}
$$

where

$$
h(k)= \begin{cases}-\mathrm{i} \mathrm{e}^{\mathrm{i} \alpha} f(k), & k \in \gamma_{d}, \\ -\mathrm{i} \mathrm{e}^{\mathrm{i} \alpha} \bar{f}^{-1}(\bar{k}), & k \in \overline{\gamma_{d}} .\end{cases}
$$

To find the solution of this RH problem, let us use the function

$$
w(k)=\sqrt{(k-E)(k-\bar{E})(k-d)(k-\bar{d})} .
$$

Since

we have as in $\S 5.4 .4$ :

$$
\left[\frac{\log F(k)}{w(k)}\right]_{+}-\left[\frac{\log F(k)}{w(k)}\right]_{-}=\frac{\log \left[h(k) \delta^{-2}(k)\right]}{w_{+}(k)}, \quad k \in \gamma_{d} \cup \overline{\gamma_{d}},
$$

$$
F(k)=\exp \left\{\frac{w(k)}{2 \pi \mathrm{i}} \int_{\gamma_{d} \cup \overline{\gamma_{d}}} \frac{\log \left[h(s) \delta^{-2}(s, \xi)\right]}{s-k} \frac{\mathrm{d} s}{w_{+}(s)}\right\} .
$$

The important difference, however, is that now the function $F(k)$ has an essential singularity at infinity. Indeed we have

$$
F(k)=F_{\infty} \mathrm{e}^{\mathrm{i} \Delta k}\left(1+\mathrm{O}\left(\frac{1}{k}\right)\right), \quad k \rightarrow \infty,
$$

where

$$
\begin{aligned}
& \Delta \equiv \Delta(\xi)=\frac{1}{2 \pi} \int_{\gamma_{d} \cup \overline{\gamma_{d}}} \log \left[h(s) \delta^{-2}(s, \xi)\right] \frac{\mathrm{d} s}{w_{+}(s)} \\
& F_{\infty}=\exp \left\{\frac{\mathrm{i}}{2 \pi} \int_{\gamma_{d} \cup \overline{\gamma_{d}}}\left(s-e_{1}\right) \log \left[h(s) \delta^{-2}(s, \xi)\right] \frac{\mathrm{d} s}{w_{+}(s)}\right\}
\end{aligned}
$$

with

$$
e_{1}=\frac{E+\bar{E}+d+\bar{d}}{2} .
$$

In order to account for this singularity, let us introduce the normalized (his a-period vanishes) Abelian integral $\omega(k)$ of the second kind with simple poles at $\infty_{ \pm}$:

$$
\omega(k)=\int_{E}^{k} \frac{z^{2}-e_{1} z+e_{0}}{w(z)} \mathrm{d} z,
$$

where $e_{1}$ is the same as in (5.18) (therefore the differential $\mathrm{d} \omega$ has no residues) and $e_{0}$ is defined from the condition

$$
\int_{\mathfrak{a}} \mathrm{d} \omega(k)=0
$$


i.e.

$$
e_{0} \int_{d}^{\bar{d}} \frac{\mathrm{d} z}{w(z)}=-\int_{d}^{\bar{d}}\left(z^{2}-e_{1} z+e_{0}\right) \frac{\mathrm{d} z}{w(z)} .
$$

The large $k$ expansion of $\omega(k)$ on the upper sheet is of the form

$$
\omega(k)=k+\omega_{\infty}(\xi)+\mathrm{O}\left(k^{-1}\right), \quad k \rightarrow \infty_{+},
$$

where

$$
\begin{aligned}
\omega_{\infty} & =\int_{E}^{\infty_{+}}\left[\frac{z^{2}-e_{1} z+e_{0}}{w(z)}-1\right] \mathrm{d} z-E \\
& \equiv \frac{1}{2}\left(\int_{E}^{\infty_{+}}+\int_{\bar{E}}^{\infty_{+}}\right)\left[\frac{z^{2}-e_{1} z+e_{0}}{w(z)}-1\right] \mathrm{d} z+b
\end{aligned}
$$

is a real function of $\xi$. The path of integration is any contour lying in the right half-plane $\operatorname{Re} k>-b$ of the upper sheet and going to infinity along the real axis. In the last identity, we have taken into account equation (5.19) and the absence of residue at infinity for $d \omega$. With the same convention about the choice of the contour of integration as in $§ 5.5 .1$ for the case of the Abelian integral $g(k)$ (the contour of integration does not intersect the augmented contour $\left.\gamma_{d} \cup \overline{\gamma_{d}} \cup[d, \bar{d}] \cup(-b+\mathrm{i} \infty, E) \cup(\bar{E},-b-\mathrm{i} \infty)\right)$, we see that the Abelian integral $\omega(k)$ satisfies similar jump relations:

$$
\begin{array}{ll}
\omega_{+}(k)+\omega_{-}(k)=0, & k \in \gamma_{d} \cup \overline{\gamma_{d}} ; \\
\omega_{+}(k)-\omega_{-}(k)=B_{\omega}, & k \in[d, \overline{d]} ; \\
\omega_{+}(k)-\omega_{-}(k)=0, \quad k \in(-b+\mathrm{i} \infty, E) \cup(\bar{E},-b-\mathrm{i} \infty) .
\end{array}
$$

Here, $B_{\omega}$ is the $\mathfrak{b}$-period of the integral $\omega(k)$ :

$$
B_{\omega}=\int_{b} \mathrm{~d} \omega=2 \int_{E}^{d} \frac{z^{2}-e_{1} z+e_{0}}{w(z)} \mathrm{d} z=\left(\int_{E}^{d}+\int_{\bar{E}}^{\bar{d}}\right) \frac{z^{2}-e_{1} z+e_{0}}{w(z)} \mathrm{d} z,
$$

where the last equation follows again from (5.19) and from the absence of residue at infinity. This equation explicitly indicates that $\operatorname{Im} B_{\omega}=0$. Let us now pass from the function $F(k)$ to the function

$$
\hat{F}(k):=F(k) \mathrm{e}^{-\mathrm{i} \Delta \omega(k)} .
$$

This function has no more essential singularity at $k=\infty$. Indeed

$$
\hat{F}(\infty, \xi)=\exp (\mathrm{i} \phi(\xi)),
$$

where

$$
\phi(\xi)=\frac{1}{2 \pi} \int_{\gamma_{d} \cup \gamma_{d}}\left(s-e_{1}\right) \log \left[-\mathrm{ie}^{\mathrm{i} \alpha} f(s) \delta^{-2}(s, \xi)\right] \frac{\mathrm{d} s}{w_{+}(s)}-\Delta \omega_{\infty} .
$$

The function $\hat{F}(k)$ has the same jumps as $F(k)$ across the arcs $\gamma_{d}$ and $\bar{\gamma}_{\bar{d}}$, and an extra jump across the interval $[d, \bar{d}]$. Indeed, we have

$$
\frac{\hat{F}_{+}(k)}{\hat{F}_{-}(k)}=\mathrm{e}^{-\mathrm{i} \Delta B_{\omega}} .
$$


5.5.3. Let us now make the last step

$$
M^{(3)}(x, t ; k) \rightsquigarrow M^{(4)}(x, t ; k),
$$

consisting in opening lenses around the contours $\gamma_{d}$ and $\overline{\gamma_{d}}$. In this step we use the function $\hat{F}(k)$ in place of $F(k)$ when performing the $N_{\text {up }}-N_{\text {low }}$ factorizations of the jump matrix $J^{(3)}(x, t ; k)$ and when defining the matrix $M^{(4)}(x, t ; k)$. This step brings us to a one-gap model problem:

\section{One-gap model RH-problem.}

$$
M_{-}^{\bmod }(x, t ; k)=M_{+}^{\bmod }(x, t ; k) J^{\bmod }(k), \quad k \in \gamma_{d} \cup \bar{\gamma}_{\bar{d}} \cup[d, \bar{d}],
$$

with jump matrix:

$$
J^{\bmod }(k)=\left\{\begin{array}{cl}
\left(\begin{array}{cc}
0 & \mathrm{ie}^{\mathrm{i} \alpha} \\
\mathrm{ie}^{-\mathrm{i} \alpha} & 0
\end{array}\right), & k \in \gamma_{d} \cup \overline{\gamma_{d}}, \\
\left(\begin{array}{cc}
\mathrm{e}^{-\mathrm{i} t B_{g}-\mathrm{i} \Delta B_{\omega}} & 0 \\
0 & \mathrm{e}^{\mathrm{i} t B_{g}+\mathrm{i} \Delta B_{\omega}}
\end{array}\right), & k \in[d, \bar{d}],
\end{array}\right.
$$

and with the asymptotic condition at $k=\infty$ :

$$
M^{\bmod }(x, t ; \infty)=I .
$$

Note the difference with the "preliminary" matrix $J^{\text {mod }}$ across the segment $[d, \bar{d}]$ indicated in (5.16). The rigorous asymptotic statement needs, of course, an analysis of the relevant parametrix in the neighborhoods of the end points. The local representation of $g(k)$ at $E$ and $\bar{E}$ is characterized by a square root type behavior:

$$
\begin{aligned}
& g(k) \sim \sqrt{k-E}+\text { const, at } E, \\
& g(k) \sim \sqrt{k-\bar{E}}+\text { const, at } \bar{E} .
\end{aligned}
$$

This means that the associated local model RH problems are solvable in terms of Bessel functions near $E$ and $\bar{E}$ (see again [9]). Similarly, the local representation of $g(k)$ at $d$ and $\bar{d}$ exhibits a 3/2-root type behavior:

$$
\begin{aligned}
& g(k) \sim(k-d)^{3 / 2}+\text { const, at } d, \\
& g(k) \sim(k-\bar{d})^{3 / 2}+\text { const, at } \bar{d} .
\end{aligned}
$$

Hence, near $d$ and $\bar{d}$, the associated local model RH problems are solvable in terms of Airy functions near $d$ and $\bar{d}$ (see again [10]). The resulting estimate for the matrix function $\left.M^{(4)}(x, t ; k)\right)$ is

$$
M^{(4)}(x, t ; k)=\left(I+\mathrm{O}\left(t^{-1 / 2}\right)\right) M^{\bmod }(x, t ; k) .
$$

It is worth mentioning, that the error term of order $t^{-1 / 2}$ comes from the contribution of the stationary phase point $\mu(\xi)$. 
5.5.4. The model problem (5.22) can be solved in terms of elliptic theta functions. For this purpose let us introduce the necessary ingredients. Consider the elliptic (two band) Riemann surface of

$$
w(k)=\sqrt{(k-E)(k-\bar{E})(k-d(\xi))(k-\bar{d}(\xi))},
$$

where the branch points $d(\xi)$ and $\bar{d}(\xi)$ depend on $\xi$. Let

$$
U(k)=\frac{1}{c} \int_{E}^{k} \frac{\mathrm{d} z}{w(z)}
$$

be the normalized Abelian integral, i.e., its a-period is equal to one, which means:

$$
c=2 \int_{\bar{d}}^{d} \frac{\mathrm{d} z}{w(z)} .
$$

Then,

$$
\tau:=\frac{2}{c} \int_{E}^{d} \frac{\mathrm{d} z}{w(z)}
$$

with $\operatorname{Im} \tau>0$. Furthermore, the following relations are valid

$$
\begin{array}{ll}
U_{+}(k)+U_{-}(k)=0, & k \in \gamma_{d} ; \\
U_{+}(k)+U_{-}(k)=-1, & k \in \overline{\gamma_{d}} ; \\
U_{+}(k)-U_{-}(k)=\tau, & k \in[d, \bar{d}] .
\end{array}
$$

The next ingredient is the new function $\nu(k)$ defined by

$$
\nu(k)^{4}=\frac{(k-E)(k-d(\xi))}{(k-\bar{E})(k-\bar{d}(\xi))}
$$

and by its asymptotic behavior as $k \rightarrow \infty, k \notin \gamma_{d} \cup \overline{\gamma_{d}}$ :

$$
\nu(k)=1+\frac{a+d_{2}(\xi)}{2 \mathrm{i} k}+\mathrm{O}\left(k^{-2}\right) .
$$

For the function $\nu(k)$ we have cuts along the contours $\gamma_{d}, \overline{\gamma_{d}}$ and $\nu_{-}(k)=\mathrm{i} \nu_{+}(k)$ along this cuts. If

$$
E_{0}=\frac{E d(\xi)-\bar{E} \bar{d}(\xi)}{E-\bar{E}+d-\bar{d}(\xi)}=\frac{a d_{1}(\xi)-b d_{2}(\xi)}{a+d_{2}(\xi)},
$$

which lies in the interval $[-b,-b+a / \sqrt{2}]$, then

$$
\nu\left(E_{0}\right)-\frac{1}{\nu\left(E_{0}\right)}=0, \quad \nu\left(E_{0}\right)+\frac{1}{\nu\left(E_{0}\right)} \neq 0 .
$$

The last ingredient is the theta function with $\operatorname{Im} \tau=\operatorname{Im} \tau(\xi)>0$ :

$$
\theta_{3}(z)=\sum_{m \in \mathbb{Z}} \mathrm{e}^{\pi \mathrm{i} \tau m^{2}+2 \pi \mathrm{i} m z}
$$

which has the following properties

$$
\theta_{3}(-z)=\theta_{3}(z), \quad \theta_{3}(z+1)=\theta_{3}(z), \quad \theta_{3}(z+\tau)=\mathrm{e}^{-\pi \mathrm{i} \tau-2 \pi \mathrm{i} z} \theta_{3}(z) .
$$


Now introduce the matrix $\Theta(k)=\Theta(t, \xi ; k)$ with entries:

$$
\begin{aligned}
\Theta_{11}(k) & =\frac{1}{2}\left[\nu(k)+\frac{1}{\nu(k)}\right] \frac{\theta_{3}\left[U(k)+U\left(E_{0}^{-}\right)-1 / 2-\tau / 2+B_{g} t / 2 \pi+B_{\omega} \Delta / 2 \pi\right]}{\theta_{3}\left[U(k)+U\left(E_{0}^{-}\right)-1 / 2-\tau / 2\right]} \\
\Theta_{12}(k) & =\frac{\mathrm{e}^{\mathrm{i} \alpha}}{2}\left[\nu(k)-\frac{1}{\nu(k)}\right] \frac{\theta_{3}\left[U(k)-U\left(E_{0}^{-}\right)+1 / 2+\tau / 2-B_{g} t / 2 \pi-B_{\omega} \Delta / 2 \pi\right]}{\theta_{3}\left[U(k)-U\left(E_{0}^{-}\right)+1 / 2+\tau / 2\right]} \\
\Theta_{21}(k) & =\frac{\mathrm{e}^{-\mathrm{i} \alpha}}{2}\left[\nu(k)-\frac{1}{\nu(k)}\right] \frac{\theta_{3}\left[U(k)-U\left(E_{0}^{-}\right)-1 / 2-\tau / 2+B_{g} t / 2 \pi+B_{\omega} \Delta / 2 \pi\right]}{\theta_{3}\left[U(k)-U\left(E_{0}^{-}\right)-1 / 2-\tau / 2\right]} \\
\Theta_{22}(k) & =\frac{1}{2}\left[\nu(k)+\frac{1}{\nu(k)}\right] \frac{\theta_{3}\left[U(k)+U\left(E_{0}^{-}\right)+1 / 2+\tau / 2-B_{g} t / 2 \pi-B_{\omega} \Delta / 2 \pi\right]}{\theta_{3}\left[U(k)+U\left(E_{0}^{-}\right)+1 / 2+\tau / 2\right]}
\end{aligned}
$$

where $E_{0}^{-}$is the preimage of $E_{0}$ on the second sheet of the Riemann surface. This function is analytic on the first sheet of the Riemann surface cut along $\gamma_{d} \cup \overline{\gamma_{d}} \cup[d, \bar{d}]$, where it satisfies the jump conditions (5.22b) of the model RH problem (5.22a). Then the solution of this problem (5.22) is given by

$$
M^{\bmod }(x, t ; k)=\Theta^{-1}(x, t ; \infty) \Theta(x, t ; k) .
$$

As in $\S 5.4 .5$, for the plane wave region,

$$
\begin{aligned}
q(x, t) & =2 \mathrm{i} m_{12}(x, t) \\
& =2 \mathrm{ie}^{2 \mathrm{i} t g_{\infty}(\xi)} m_{12}^{(1)}(x, t) \\
& =2 \mathrm{ie}^{2 \mathrm{i} t g_{\infty}(\xi)} m_{12}^{(2)}(x, t) \\
& =2 \mathrm{ie}^{2 \mathrm{i} t g_{\infty}(\xi)} m_{12}^{(3)}(x, t)+\mathrm{O}\left(t^{-1 / 2}\right) \\
& =2 \mathrm{ie}^{2 \mathrm{i} t g_{\infty}(\xi)} m_{12}^{(4)}(x, t) \hat{F}^{-2}(\infty)+\mathrm{O}\left(t^{-1 / 2}\right) \\
& =2 \mathrm{ie}^{2 \mathrm{i} t g_{\infty}(\xi)} m_{12}^{\bmod }(x, t) \hat{F}^{-2}(\infty)+\mathrm{O}\left(t^{-1 / 2}\right) .
\end{aligned}
$$

Take into account that

$$
2 \mathrm{i} m_{12}^{\bmod }(x, t)=\left[a+d_{2}(\xi)\right] \mathrm{e} \mathrm{e}^{\mathrm{i} \alpha} \frac{\theta_{3}\left[B_{g} t / 2 \pi++B_{\omega} \Delta / 2 \pi-U\left(\infty_{+}\right)+U_{0}\right]}{\theta_{3}\left[B_{g} t / 2 \pi+B_{\omega} \Delta / 2 \pi+U\left(\infty_{+}\right)+U_{0}\right]} \frac{\theta_{3}\left[U\left(\infty_{+}\right)+U_{0}\right]}{\theta_{3}\left[U\left(\infty_{+}\right)-U_{0}\right]},
$$

and $\hat{F}^{-2}(\infty)=\mathrm{e}^{-2 \mathrm{i} \phi(\xi)}$, we get the asymptotics of the solution of the IBV problem (1.1) in the region $b-a \sqrt{2}<\xi<b$.

Theorem 5 (elliptic region, $b-a \sqrt{2}<\xi<b$ ). Suppose that all conditions of Theorem 1 and conditions \#1, \#2, \#3 are satisfied.

Then in the region $b-a \sqrt{2}<\xi<b$ the solution (4.2) of the IBV problem takes the form of a modulated elliptic wave:

$$
q(x, t)=[a+\operatorname{Im} d(\xi)] \mathrm{e}^{\mathrm{i} \alpha} \frac{\theta_{3}\left(B_{g} t / 2 \pi+B_{\omega} \Delta / 2 \pi+V_{-}\right) \theta_{3}\left(V_{+}\right)}{\theta_{3}\left(B_{g} t / 2 \pi+B_{\omega} \Delta / 2 \pi+V_{+}\right) \theta_{3}\left(V_{-}\right)} \mathrm{e}^{2 \mathrm{i} g_{\infty}(\xi) t-2 \mathrm{i} \phi(\xi)}+\mathrm{O}\left(t^{-1 / 2}\right) .
$$


Here, $B_{g}, B_{\omega}, \Delta$ are functions of the slow variable $\xi=\frac{x}{4 t}$ defined by (5.13), (5.21), (5.17), respectively, and

$$
\begin{aligned}
& V_{+}=U\left(E_{0}^{-}\right)-\frac{1}{2}-\frac{\tau(\xi)}{2}+U\left(\infty_{+}\right), \\
& V_{-}=U\left(E_{0}^{-}\right)-\frac{1}{2}-\frac{\tau(\xi)}{2}-U\left(\infty_{+}\right) .
\end{aligned}
$$

Furthermore,

$$
\theta_{3}(z)=\sum_{m \in \mathbb{Z}} \mathrm{e}^{\pi \mathrm{i} \tau m^{2}+2 \pi \mathrm{i} m z}
$$

is the theta function of invariant $\tau=\tau(\xi), \operatorname{Im} \tau>0$, defined in (5.24) and

$$
g_{\infty}(\xi)=2\left(\int_{E}^{\infty}+\int_{\bar{E}}^{\infty}\right)\left[(z-\mu(\xi)) \sqrt{\frac{(z-d(\xi))(z-\bar{d}(\xi))}{(z-E)(z-\bar{E})}}-(z+\xi)\right] \mathrm{d} z+2 a^{2}-2 b^{2}+4 b \xi
$$

is a regularization of the phase function $g(k)$. Finally, the phase shift is given by

where

$$
\phi(\xi)=\frac{1}{2 \pi} \int_{\gamma_{d} \cup \overline{\gamma_{d}}}\left(s-e_{1}-\omega_{\infty}\right) \log \left[h(s) \delta^{-2}(s, \xi)\right] \frac{\mathrm{d} s}{w_{+}(s)}
$$

$$
\begin{aligned}
& h(k)= \begin{cases}-\mathrm{ie}^{\mathrm{i} \alpha} f(k), & k \in \gamma_{d}, \\
-\mathrm{i}^{\mathrm{i} \alpha} \bar{f}^{-1}(\bar{k}), & k \in \overline{\gamma_{d}},\end{cases} \\
& \delta(k)=\exp \left\{\frac{1}{2 \pi \mathrm{i}} \int_{-\infty}^{\mu(\xi)} \frac{\log \left(1+|r(s)+c(s)|^{2}\right) \mathrm{d} s}{s-k}\right\}, \quad k \in \mathbb{C} \backslash(-\infty, \mu(\xi)]
\end{aligned}
$$

and $e_{1}=e_{1}(\xi), \Delta=\Delta(\xi), \omega_{\infty}=\omega_{\infty}(\xi)$ and $\mu(\xi)$ are defined by (5.18), (5.17), (5.20) and (5.15), respectively. The spectral functions $c(k)$ and $r(k)$ are defined by the initial and boundary data, see (2.14) and (2.15), respectively, and $f(k)=c_{-}(k)-c_{+}(k)$ is the jump of $c(k)$, see $(2.21)$.

Remark 5. It is easy to verify that for $\xi=\xi_{0}$ the elliptic solution (5.25) coincides with the solution (5.11) in the plane wave region.

Remark 6. If $b^{2}=2 a^{2}$, i.e., $\xi_{0}=0$ then the asymptotic behavior of the solution is only described by elliptic functions with modulated parameter, and the plane wave region disappears.

Acknowledgments. The authors thank Percy Deift and Dmitry Shepelsky for useful discussions, and Chunxiong Zheng for numeric simulations of the problem. The second author thanks also John Elgin and John Gibbons. His work was supported in part by NSF grant DMS-0401009.

\section{REFERENCES}

[1] M. J. Ablowitz and H. Segur, Solitons and the inverse scattering transform, SIAM Studies in Applied Mathematics, vol. 4, Society for Industrial and Applied Mathematics (SIAM), Philadelphia, Pa., 1981.

[2] A. Boutet de Monvel, A. S. Fokas, and D. Shepelsky, The mKdV equation on the half-line, J. Inst. Math. Jussieu 3 (2004), no. 2, 139-164. 
[3] A. Boutet de Monvel, A. R. Its, and V. Kotlyarov, Long-time asymptotics for the focusing NLS equation with time-periodic boundary condition, C. R. Math. Acad. Sci. Paris 345 (2007), no. 11, 615-620.

[4] A. Boutet de Monvel and V. Kotlyarov, Scattering problem for the Zakharov-Shabat equations on the semi-axis, Inverse Problems 16 (2000), no. 6, 1813-1837.

[5] - Generation of asymptotic solitons of the nonlinear Schrödinger equation by boundary data, J. Math. Phys. 44 (2003), no. 8, 3185-3215. Integrability, topological solitons and beyond.

[6] The Focusing Nonlinear Schrödinger Equation on the Quarter Plane with Time-Periodic Boundary Condition: a Riemann-Hilbert approach, J. Inst. Math. Jussieu 6 (2007), no. 4, 579-611.

[7] R. Buckingham and S. Venakides, Long-Time Asymptotics of the Nonlinear Schrödinger Equation Shock Problem, Comm. Pure Appl. Math. 60 (2007), no. 9, 1349-1414.

[8] P. A. Deift, A. R. Its, and X. Zhou, Long-time asymptotics for integrable nonlinear wave equations, Important developments in soliton theory, Springer Ser. Nonlinear Dynam., Springer, Berlin, 1993, pp. 181-204.

[9] A Riemann-Hilbert approach to asymptotic problems arising in the theory of random matrix models, and also in the theory of integrable statistical mechanics, Ann. of Math. (2) 146 (1997), no. 1, 149-235.

[10] P. Deift, T. Kriecherbauer, K. T.-R. McLaughlin, S. Venakides, and X. Zhou, Uniform asymptotics for polynomials orthogonal with respect to varying exponential weights and applications to universality questions in random matrix theory, Comm. Pure Appl. Math. 52 (1999), no. 11, 1335-1425.

[11] P. Deift and X. Zhou, A steepest descent method for oscillatory Riemann-Hilbert problems, Bull. Amer. Math. Soc. (N.S.) 26 (1992), no. 1, 119-123.

[12] _ A steepest descent method for oscillatory Riemann-Hilbert problems. Asymptotics for the MKdV equation, Ann. of Math. (2) 137 (1993), no. 2, 295-368.

[13] A. S. Fokas and A. R. Its, The linearization of the initial-boundary value problem of the nonlinear Schrödinger equation, SIAM J. Math. Anal. 27 (1996), no. 3, 738-764.

[14] A. S. Fokas, A. R. Its, and L.-Y. Sung, The nonlinear Schrödinger equation on the half-line, Nonlinearity 18 (2005), no. 4, 1771-1822.

[15] A. S. Fokas and C. R. Menyuk, Integrability and self-similarity in transient stimulated Raman scattering, J. Nonlinear Sci. 9 (1999), no. 1, 1-31.

[16] D. J. Kaup and H. Steudel, Virtual solitons and the asymptotics of second harmonic generation, Inverse Problems 17 (2001), no. 4, 959-970. Special issue to celebrate Pierre Sabatier's 65th birthday (Montpellier, 2000).

[17] V. E. Zakharov and A. B. Shabat, Exact theory of two-dimensional self-focusing and one-dimensional self-modulation of waves in nonlinear media, Ž. Èksper. Teoret. Fiz. 61 (1971), no. 1, 118-134 (Russian, with English summary); English transl., Soviet Physics JETP 34 (1972), no. 1, 62-69.

*imJ, Case 7012, Université Paris 7, 2 Place Jussieu, 75251 Paris, France

${ }^{\dagger}$ Indiana University - Purdue University Indianapolis, IN, USA

${ }^{\ddagger}$ Math. Div., Inst. B. Verkin, 47 Lenin Avenue, 61103 Kharkiv, Ukraine 